\title{
Composing alternatives
}

\author{
Ivano Ciardelli ${ }^{1}$ • Floris Roelofsen ${ }^{1}$. \\ Nadine Theiler ${ }^{1}$
}

(C) The Author(s) 2016. This article is published with open access at Springerlink.com

\begin{abstract}
There is a prominent line of work in natural language semantics, rooted in the work of Hamblin, in which the meaning of a sentence is not taken to be a single proposition, but rather a set of propositions - a set of alternatives. This allows for a more fine-grained view on meaning, which has led to improved analyses of a wide range of linguistic phenomena. However, this approach also faces a number of problems. We focus here on two of these, in our view the most fundamental ones. The first has to do with how meanings are composed, i.e., with the type-theoretic operations of function application and abstraction; the second has to do with how meanings are compared, i.e., the notion of entailment. Our aim is to reconcile what we take to be the essence of Hamblin's proposal with the more orthodox type-theoretic framework rooted in the work of Montague in such a way that both the explanatory utility of the former and the solid formal foundations of the latter are preserved. Our proposal builds on insights from recent work on inquisitive semantics, and it also contributes to the further development of this framework by specifying how the inquisitive meaning of a sentence may be built up compositionally.
\end{abstract}

This paper integrates and extends ideas and results from Theiler (2014) and Ciardelli and Roelofsen (2015). We are grateful to two anonymous reviewers for their useful feedback, to Andreas Haida, Reinhard Muskens, Wataru Uegaki, and Yimei Xiang for helpful comments on earlier presentations of this material, and especially to Maria Aloni, Lucas Champollion, Liz Coppock, Donka Farkas, Jeroen Groenendijk, Edgar Onea, and Anna Szabolcsi for extensive discussion. Financial support from the Netherlands Organization for Scientific Research (NWO) is gratefully acknowledged.

Floris Roelofsen

floris.roelofsen@gmail.com

1 Institute for Logic, Language, and Computation, University of Amsterdam, P. O. Box 94242, 1090 GE Amsterdam, The Netherlands 
Keywords Alternative semantics - Inquisitive semantics - Type-theoretic semantics . Compositionality

\section{Introduction}

There is a prominent and fruitful line of work in natural language semantics which deviates from the standard Montagovian approach (Montague 1970, 1973) in that it takes the semantic value of an expression to be a set of objects in the expression's usual domain of interpretation, rather than a single object. For instance, the semantic value of a complete sentence is not taken to be a proposition but a set of propositions, the semantic value of an individual-denoting expression is not taken to be an individual but a set of individuals, and so on. In this framework, the elements of the semantic value of an expression are called alternatives, and the framework itself is referred to as alternative semantics. A range of linguistic phenomena have received insightful analyses in alternative semantics, including questions (Hamblin 1973), focus (Rooth 1985), indeterminate pronouns (Shimoyama 2001; Kratzer and Shimoyama 2002), indefinites (Kratzer and Shimoyama 2002; Menéndez-Benito 2005; Aloni 2007), and disjunction (Simons 2005; Alonso-Ovalle 2006; Aloni 2007). ${ }^{1}$ While this wealth of applications shows that alternatives are a useful tool in the semantic analysis of natural language, the move from the orthodox type-theoretic framework to an alternative-based one also raises some fundamental issues. In this paper, we will be concerned with two of these issues, in our opinion the most basic ones. The first issue, which we will refer to as the compositionality issue, has to do with the fact that in alternative semantics, meanings can no longer be composed by means of the standard type-theoretic operations of function application and abstraction. The second, which we will refer to as the entailment issue, has to do with the fact that meanings in alternative semantics can no longer be compared by means of the standard type-theoretic notion of entailment. Both problems concern very fundamental features of the semantic framework, and moreover, as we shall see, neither of them has a straightforward solution.

We will examine why these problems arise. Our diagnosis will be that it is not the presence of alternatives per se that is to be held responsible, but rather some specific features of the architecture of alternative semantics. For two such features we will argue that they are not essential for the utility of the framework, and we will show how making different architectural choices results in a framework in which the observed problems do not arise.

While the general aim of this paper is to reconcile alternative-based semantic theories developed in the Hamblin tradition with the more orthodox type-theoretic framework rooted in Montague's work, it also contributes to a more recent and more specific line of work, namely that of developing the framework of inquisitive semantics (Ciardelli et al. 2013, 2015, among others). Namely, while previous work on inquisitive semantics has laid out a formal notion of sentence meaning that is more fine-grained than the standard truth-conditional notion—comprising both informative

\footnotetext{
${ }^{1}$ We will concentrate here on the role of alternatives at the level of ordinary semantic values, not at the level of focus semantic values (Rooth 1985). We will only briefly discuss the treatment of focus in footnote 30 .
} 


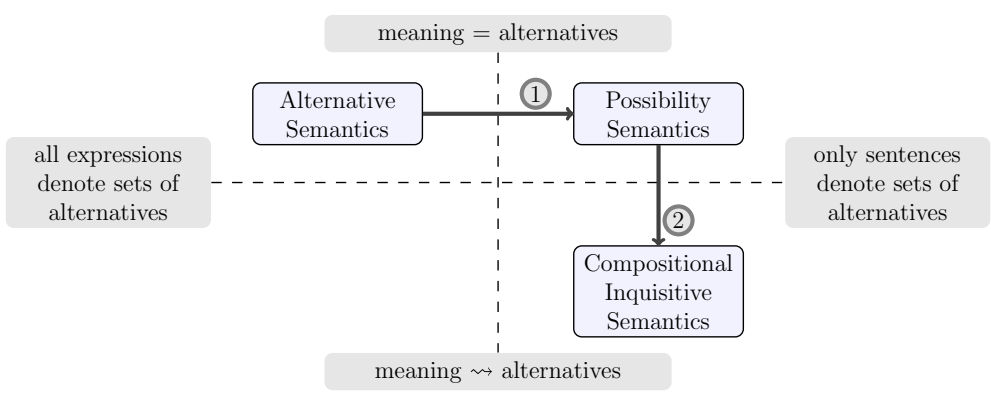

Fig. 1 Overview of the paper

and inquisitive content - it has not been specified in much detail how the meaning of a sentence, construed in this more fine-grained way, is to be composed from the meanings of the words that it consists of. This open issue is addressed here; the framework that we will end up with is a fully compositional inquisitive semantics.

We will arrive at this result in two steps, which are summarized in Fig. 1. Our point of departure is a Hamblin-style alternative semantics (in the upper left quadrant of the figure). In the first step, marked as 1 in the diagram, we will give up a certain feature of alternative semantics, namely the assumption that all expressions denote sets of objects in their usual domain of interpretation. Rather, we will assume that this is only the case for sentences. This step will lead us to a framework that we call possibility semantics, in which one of the issues mentioned above, namely the compositionality issue, is avoided. This means that, in this framework, meanings can be composed by means of the standard type-theoretic operations.

In the second step, marked as 2 in the diagram, we will move from possibility semantics to a compositional inquisitive semantics. This will amount to giving up another feature of alternative semantics, namely the assumption that the meaning of a sentence is identical with the set of alternatives it generates. Instead, we will adopt the weaker assumption that the meaning of a sentence determines the set of alternatives that it generates, without necessarily being identical to it. This second step solves the entailment issue: in the resulting framework meanings can again be compared by means of the standard type-theoretic notion of entailment. Thus, in the compositional inquisitive semantics that we propose neither of the initial problems arises.

The paper has a straightforward structure: Sect. 2 is concerned with the compositionality issue (step (1), Sect. 3 with the entailment issue (step (2), and Sect. 4 concludes.

\section{Compositionality}

In the standard type-theoretic semantic framework, the semantic value of an expression $\boldsymbol{\alpha}$ of type $\tau$ (notation: $\boldsymbol{\alpha}: \tau$ ) relative to an assignment $g$ is an object $\llbracket \boldsymbol{\alpha} \rrbracket_{g}$ in the corresponding domain $D_{\tau}$, where the basic types $e, t$, and $s$ correspond to primitive domains of individuals, truth-values and possible worlds, respectively, and a derived type $\langle\sigma, \tau\rangle$ corresponds to the domain $D_{\langle\sigma, \tau\rangle}=\left\{f \mid f: D_{\sigma} \rightarrow D_{\tau}\right\}$ of functions from 
objects of type $\sigma$ to objects of type $\tau$. This setup allows us to compose meanings through the basic type-theoretic operations of function application and abstraction:

(1) Function Application: if $\boldsymbol{\alpha}:\langle\sigma, \tau\rangle$ and $\boldsymbol{\beta}: \sigma$ then $\llbracket \boldsymbol{\alpha}(\boldsymbol{\beta}) \rrbracket_{g}=\llbracket \boldsymbol{\alpha} \rrbracket_{g}\left(\llbracket \boldsymbol{\beta} \rrbracket_{g}\right) \in D_{\tau}$

(2) Abstraction: if $\alpha: \tau$ and $\boldsymbol{x}: \sigma$ then $\llbracket \lambda \boldsymbol{x} . \boldsymbol{\alpha} \rrbracket_{g}$ is the function mapping any $x \in D_{\sigma}$ to $\llbracket \boldsymbol{\alpha} \rrbracket g[x \mapsto x]$

In the meta-language we will use $\lambda x \cdot \llbracket \boldsymbol{\alpha} \rrbracket_{g[\boldsymbol{x} \mapsto x]}$ as a shorthand description of this function. ${ }^{2}$

By contrast, in alternative semantics the semantic value $\llbracket \boldsymbol{\alpha} \rrbracket_{g}$ of an expression $\boldsymbol{\alpha}: \tau$ is no longer a single object in $D_{\tau}$, but rather a set of such objects: $\llbracket \boldsymbol{\alpha} \rrbracket_{g} \subseteq D_{\tau}{ }^{3}$ As a consequence, meanings can no longer be composed by means of the standard type-theoretic operations. Let us see why.

\subsection{Composition in alternative semantics}

\subsubsection{Function application}

First consider the operation of function application. Suppose $\boldsymbol{\alpha}$ is an expression of type $\langle\sigma, \tau\rangle$ and $\boldsymbol{\beta}$ an expression of type $\sigma$. In alternative semantics, we have that $\llbracket \boldsymbol{\alpha} \rrbracket_{g} \subseteq D_{\langle\sigma, \tau\rangle}$ and $\llbracket \boldsymbol{\beta} \rrbracket_{g} \subseteq D_{\sigma}$. Now suppose we want to compute the meaning of $\boldsymbol{\alpha}(\boldsymbol{\beta})$. We can no longer obtain $\llbracket \boldsymbol{\alpha}(\boldsymbol{\beta}) \rrbracket_{g}$ by simply applying $\llbracket \boldsymbol{\alpha} \rrbracket_{g}$ to $\llbracket \boldsymbol{\beta} \rrbracket_{g}$, because $\llbracket \alpha \rrbracket_{g}$ is not a single function from $D_{\sigma}$ to $D_{\tau}$, but a set of such functions. Thus, the type-theoretic rule of function application cannot be used to compute $\llbracket \boldsymbol{\alpha}(\boldsymbol{\beta}) \rrbracket_{g}$.

Instead, $\llbracket \boldsymbol{\alpha} \rrbracket_{g}$ is now a set of functions from objects of type $\sigma$ to objects of type $\tau$. Since $\llbracket \boldsymbol{\beta} \rrbracket_{g}$ is a set of objects of type $\sigma$, what we can naturally do is apply each function $f \in \llbracket \boldsymbol{\alpha} \rrbracket_{g}$ to each object $d \in \llbracket \boldsymbol{\beta} \rrbracket_{g}$. The set of all objects $f(d)$ obtained in this way is a subset of $D_{\tau}$, and thus a suitable semantic value for $\boldsymbol{\alpha}(\boldsymbol{\beta})$. This operation, known as pointwise function application, is indeed taken to be the fundamental composition rule in alternative semantics.

(3) Pointwise function application: if $\boldsymbol{\alpha}:\langle\sigma, \tau\rangle$ and $\boldsymbol{\beta}: \sigma$ then $\boldsymbol{\alpha}(\boldsymbol{\beta}): \tau$ and $\llbracket \boldsymbol{\alpha}(\boldsymbol{\beta}) \rrbracket_{g}=\left\{f(d) \mid f \in \llbracket \boldsymbol{\alpha} \rrbracket_{g}\right.$ and $\left.d \in \llbracket \boldsymbol{\beta} \rrbracket_{g}\right\}$

However, this rule has an important drawback. In computing the meaning of a complex expression $\boldsymbol{\alpha}(\boldsymbol{\beta})$ using pointwise function application, the functor $\boldsymbol{\alpha}$ only has access

\footnotetext{
2 Our general typographic convention is to use boldface for expressions in the object language ('logical form'), and the standard font for meta-language descriptions of semantic objects.

3 In some work on alternative semantics, the types that are assigned to expressions are systematically adapted: expressions that are usually taken to be of type $\tau$ are now rather taken to be of type $\langle\tau, t\rangle$ (see, e.g., Shan 2004; Novel and Romero 2010). The usual correspondence between the type of an expression and its semantic value is then preserved. In other work, the usual types are preserved: expressions that are usually taken to be of type $\tau$ are still taken to be of type $\tau$ (see, e.g., Kratzer and Shimoyama 2002; Alonso-Ovalle 2006). In this case, the correspondence between the type of an expression and its semantic value changes: the semantic value of an expression of type $\tau$ is no longer a single object in $D_{\tau}$, but rather a set of such objects. The choice between these two options seems immaterial; for concreteness we assume the second, but our arguments do not hinge on this assumption.
} 
to each alternative for $\boldsymbol{\beta}$ in isolation; it does not have access to the whole set at once. This is problematic because, in fact, many functors in natural language $d o$ need access to the whole set of alternatives introduced by their argument at once. Take for instance negation. The standard treatment of sentential negation in alternative semantics is as follows (see, e,g., Kratzer and Shimoyama 2002):

$$
\llbracket \operatorname{not} \boldsymbol{\beta} \rrbracket_{g}=\left\{\overline{U \llbracket \boldsymbol{\beta} \rrbracket_{g}}\right\}
$$

where $\overline{\cup \llbracket \boldsymbol{\beta} \rrbracket_{g}}$ denotes the set-theoretic complement of $\bigcup \llbracket \boldsymbol{\beta} \rrbracket_{g}$

To determine $\overline{U \boldsymbol{\beta} \rrbracket_{g}}$, not clearly needs access to all the alternatives for $\boldsymbol{\beta}$ at once. This result is impossible to obtain by associating negation with a set of objects $\llbracket$ not $\rrbracket_{g} \in$ $D_{\langle s t, s t\rangle}$ and letting them combine with the alternatives for $\boldsymbol{\beta}$ by pointwise function application. Thus, negation needs to be treated syncategorematically, that is, by means of a tailor-made rule in the grammar.

This problem is not confined to a few exceptional cases: in fact, the class of operators that need access to the whole set of alternatives for their argument includes virtually all operators that are interesting from an alternative semantics perspective: modals (e.g., Simons 2005; Aloni 2007), conditionals (e.g., Alonso-Ovalle 2006, unconditionals (Rawlins 2008), exclusive strengthening operators (e.g., Menéndez-Benito 2005; Alonso-Ovalle 2006; Roelofsen and van Gool 2010), existential and universal closure operators (e.g., Kratzer and Shimoyama 2002), and question-embedding verbs. Adopting pointwise function application as our fundamental composition rule implies that none of these operators can be given a meaning of their own. Instead, they all have to be treated by means of tailor-made, syncategorematic composition rules. Clearly, this is undesirable: we would like our grammar to contain only a few, general composition rules, and we would like the contribution of a specific linguistic item to be derivable from its lexical meaning, based on these general rules.

\subsubsection{Abstraction}

Now let us consider abstraction. Suppose $\alpha: \tau$ contains a variable $\boldsymbol{x}: \sigma$, and suppose we want to abstract over $\boldsymbol{x}$ to obtain an expression $\lambda \boldsymbol{x} . \boldsymbol{\alpha}$ of type $\langle\sigma, \tau\rangle$. This is an operation that is often used in semantics, typically (though not exclusively) in order to deal with quantification. What semantic value should we assign to $\lambda \boldsymbol{x} . \boldsymbol{\alpha}$ ? We cannot apply the standard abstraction rule, which would identify $\llbracket \lambda \boldsymbol{x} . \boldsymbol{\alpha} \rrbracket_{g}$ with the function mapping every $x \in D_{\sigma}$ to $\llbracket \boldsymbol{\alpha} \rrbracket_{g[x \mapsto x]}$, for that would be a function from $D_{\sigma}$ to subsets of $D_{\tau}$. What we need for $\llbracket \lambda \boldsymbol{x} . \boldsymbol{\alpha} \rrbracket_{g}$ is a different object, namely, a set of functions from $D_{\sigma}$ to $D_{\tau}$, since we want that $\llbracket \lambda \boldsymbol{x} . \boldsymbol{\alpha} \rrbracket_{g} \subseteq D_{\langle\sigma, \tau\rangle}$. Thus, standard abstraction cannot be applied in alternative semantics.

What is more, there does not seem to be a straightforward way of defining a different abstraction rule for alternative semantics that would yield correct results. A natural candidate for such a rule was suggested by Hagstrom (1998) and later adopted by Kratzer and Shimoyama (2002). Shan (2004), however, pointed out that this proposal, combined with the standard techniques for quantification and binding, leads to wrong empirical predictions. He furthermore argued that it is impossible to obtain the right 
set of functions in a principled way, and that an alternative-based notion of meaning therefore calls for a variable-free approach to meaning composition (Szabolcsi 1987; Jacobson 1999), which does entirely without abstraction. Novel and Romero (2010) argue that the cases which Shan deemed problematic could in fact be dealt with by enriching the underlying type theory with a new basic type for assignments for $e$-type variables, ${ }^{4}$ and making certain assumptions about the meaning of wh-items. Charlow (2014), however, argues that Novel and Romero's strategy still encounters problems.

We will not directly enter the debate on this front, in the sense that, unlike Shan, we will not depart from lambda abstraction and, unlike Novel and Romero, we will not try to extend classical alternative semantics in a way that allows for an alternativefriendly abstraction rule. Instead, we will take a more conservative approach, and ask whether it is at all necessary to depart from the standard, i.e., non-pointwise abstraction mechanism. Indeed, we will show that it isn't, provided that the basic architecture of the framework is adapted in certain ways. In the resulting framework, the cases deemed problematic by Shan and others will turn out to be unproblematic.

\subsection{Possibility semantics}

In our view, the feature of alternative semantics that is responsible for its empirical success is the fact that sentences are taken to express sets of propositions, rather than single propositions. This yields a notion of sentence meaning that is more structured than the standard, truth-conditional notion, and this extra structure seems to play a key role in a range of linguistic phenomena.

However, alternative semantics does not just assume that sentences express sets of propositions: it goes on to assume that every expression denotes a set of objects in its usual domain of interpretation. As we have seen, this stronger assumption forces us to depart from the standard composition rules.

There does not seem to be any particular conceptual motivation for the assumption that every expression denotes a set of objects. Moreover, in linguistic applications of the framework the assumption does not seem essential, as we will show in a moment for some concrete cases. Most importantly, if we discharge this stronger assumption, then it becomes apparent that the remaining, more fundamental assumption, i.e., that sentences express sets of propositions, is perfectly compatible with the standard typetheoretic operations of meaning composition. We will demonstrate this by laying out a framework that is based on the following three assumptions:

1. the semantic value of a complete sentence is a set of propositions;

2. the semantic value of an expression of type $\tau$ is a single object in $D_{\tau}$;

3. the fundamental composition rules are the standard type-theoretic ones.

In this framework, which we will refer to as possibility semantics, it is not the compositional machinery, but rather the typing of expressions that needs to be adjusted. For instance, consider a complete sentence $\boldsymbol{\alpha}$. By assumption (1), its semantic value $\llbracket \boldsymbol{\alpha} \rrbracket_{g}$ should be a set of propositions. Moreover, by assumption (2), $\llbracket \alpha \rrbracket_{g}$ has to be an object

\footnotetext{
4 Novel and Romero attribute this strategy to Poesio (1996); a very similar approach was taken in Rooth (1985) to compose focus semantic values.
} 
in the domain $D_{\tau}$ of the corresponding type. Thus, we must take sentences to be of a type $\tau$ such that the objects in $D_{\tau}$ are sets of propositions: this is the type $\langle\langle s, t\rangle, t\rangle$, which we will abbreviate for convenience as $T .{ }^{5}$

Assuming standard syntactic structures for sentences, we can then use assumption (3) to reverse engineer the types that should be assigned to various sorts of sub-sentential expressions. For instance, the following types suggest themselves for verbs, sentential operators, and quantifiers. ${ }^{6}$

- walks : $\langle e, T\rangle$

- likes : $\langle e,\langle e, T\rangle\rangle$
- not : $\langle T, T\rangle$

- or : $\langle T,\langle T, T\rangle\rangle$
- nobody : $\langle\langle e, T\rangle, T\rangle$

- who : $\langle\langle e, T\rangle, T\rangle$

Thus, the relation between alternative semantics and possibility semantics may be represented succinctly as follows.

\section{Proposition-set semantics}

Basic assumption:

sentences denote sets of propositions

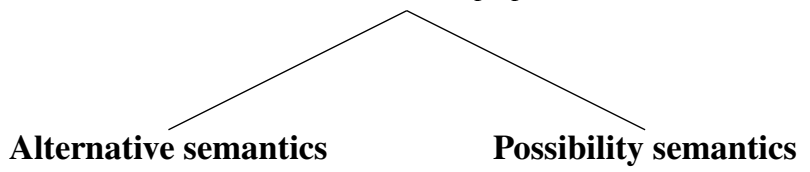

Further assumption:

all expressions denote sets

$\Downarrow$

Consequence:

composition rules need to be adapted

standard type-theoretic composition rules

$\Downarrow$

Consequence:

typing needs to be adapted

Now let us consider the actual meanings that should be assigned to expressions in possibility semantics. In alternative semantics, a basic sentence like John walks is taken to express the singleton set $\{|W j|\}$, which has as its unique element the proposition that John walks. ${ }^{7}$

$\llbracket$ John walks $\rrbracket=\{|W j|\}=\{\{w \mid j$ walks in $w\}\}$

This treatment may be adopted in possibility semantics as well. Then, using assumption (3) again, we can work backwards to infer what meanings should be

\footnotetext{
5 Strictly speaking, an object of type $\langle\langle s, t\rangle, t\rangle$ is a function from propositions to truth values. However, it is common practice to identify such a function with the set of propositions that it maps to 1 . We will discuss this in more detail below.

6 These types are the simplest options for the given items, but as usual, they are not the only available choice.

7 Throughout the paper we will assume that our logical language contains predicate symbols corresponding to the verbs and nouns in the fragment of English that we are considering (e.g., $W$ for walks) as well as individual constants corresponding to the proper names (e.g., $j$ for John). Moreover, for any expression $\varphi$ of type $t$ in our logical language, we will use $|\varphi|$ in the meta-language to denote the set of worlds where $\varphi$ holds. For instance, $|W j|$ is the set of worlds where John walks. Finally, we assume that our logical language contains the standard Boolean connectives that apply to expressions of type $t$. For instance, $|\neg W j|$ denotes the set of worlds where John does not walk.
} 
assigned to sub-sentential constituents. If we take proper names like John to denote individuals, then this procedure immediately suggests the following entry for walks:

$$
\llbracket \mathbf{w a l k s} \rrbracket=\lambda x_{e} \cdot\{|W x|\}=\lambda x_{e} \cdot\{\{w \mid x \text { walks in } w\}\}
$$

Using the same strategy, i.e., starting from the desired sentential meanings, we can infer suitable meanings for other sub-sentential constituents. Below, a small compositional fragment of English is specified, obeying assumptions 1-3. In particular, in accordance with assumption 3, the default mode of composition is function application. Items of type $\langle\langle e, T\rangle, T\rangle$ may additionally be subjected to standard quantifier raising. This fragment is not intended to provide a fully satisfactory analysis of the relevant items and constructions, but just to illustrate the compositional architecture that we propose. ${ }^{8}$

- Proper names and variables:

$$
\begin{aligned}
& \text { - } \llbracket \mathbf{J o h n} \rrbracket_{g}=j \\
& \text { - } \llbracket \boldsymbol{x} \rrbracket_{g}=g(\boldsymbol{x})
\end{aligned}
$$

- Connectives:

$$
\begin{aligned}
& \text { - } \llbracket \mathbf{o r}_{T} \rrbracket=\lambda \mathcal{P}_{T} \lambda \mathcal{Q}_{T} \cdot \mathcal{P} \cup \mathcal{Q} \\
& \text { - } \llbracket \operatorname{not}_{T} \rrbracket=\lambda \mathcal{P}_{T} \cdot\{\overline{\mathcal{P}}\}
\end{aligned}
$$

- Existential closure: $:^{10}$

$$
-\llbracket \exists \rrbracket=\lambda \mathcal{P}_{T} \cdot\{\bigcup \mathcal{P}\}
$$

A comment on our notation is in order at this point. Notice that we use set-theoretic notation mixed with type-theoretic notation to enhance readability. As is common practice, we identify a set $S \subseteq D_{\sigma}$ with its characteristic function $f_{S} \in D_{\langle\sigma, t\rangle}$, which maps any object $d \in D_{\sigma}$ to 1 just in case $d \in S$. Relying on this identification, we write, e.g., $\llbracket \mathbf{J o h n}$ walks $\rrbracket=\{|W j|\}$ instead of $\llbracket$ John walks $\rrbracket=\lambda p_{s t} .(p=|W j|)$; similarly, we write $\llbracket \mathbf{w a l k} \rrbracket=\lambda x_{e} \cdot\{|W x|\}$ instead of $\llbracket \mathbf{w a l k} \rrbracket=\lambda x_{e} \cdot \lambda p_{s t} \cdot(p=|W x|)$.

More generally, we identify a set of $n$-tuples $S \subseteq D_{\sigma_{1}} \times \ldots \times D_{\sigma_{n}}$ with the function $f_{S} \in D_{\left\langle\sigma_{1}, \ldots,\left\langle\sigma_{n}, t\right\rangle\right\rangle}$ such that for every tuple $\left\langle d_{1}, \ldots, d_{n}\right\rangle \in D_{\sigma_{1}} \times \ldots \times D_{\sigma_{n}}$, $f\left(d_{1}\right) \ldots\left(d_{n}\right)=1$ just in case $\left\langle d_{1}, \ldots, d_{n}\right\rangle \in S$. Types of the form $\left\langle\sigma_{1}, \ldots,\left\langle\sigma_{n}, t\right\rangle\right\rangle$, whose objects can be identified with sets of $n$-tuples, are known as conjoinable types (Partee and Rooth 1983).

The inclusion relation between sets and the binary operations of union and intersection can also be encoded in type theory. Given two sets of $n$-tuples $S$ and $S^{\prime}$ encoded

\footnotetext{
8 Note in particular that items with a conjunctive semantics (and, everybody) are missing from the fragment specified here. Our reasons for leaving them out here will become clear in Sect. 3, where we will argue that such items are problematic for both alternative and possibility semantics.

9 Filling in the denotation of $\llbracket$ not $\rrbracket$, we get that $\llbracket \operatorname{nobody} \rrbracket=\lambda P_{\langle e, T\rangle} \cdot\left\{\left\{w \in D_{s} \mid w \notin \bigcup P x\right.\right.$ for any $x \in$ $\left.D_{e}\right\}$.

10 The $\exists$-operator corresponds to the existential closure operator in alternative semantics (see, e.g., Kratzer and Shimoyama 2002). It takes a set of propositions and maps it to a singleton set that contains the union of these propositions, hence eliminating the structure of the original alternative set. The resulting proposition is true at a world exactly if one of the original propositions is true at that world. Hence, the $\exists$-operator has the same semantics as existential closure in alternative semantics, but, unlike in alternative semantics, it is defined categorematically.
} 
as elements of $D_{\left\langle\sigma_{1}, \ldots,\left\langle\sigma_{n}, t\right\rangle\right\rangle}$, and letting $\vec{x}$ range over tuples in $D_{\sigma_{1}} \times \cdots \times D_{\sigma_{n}}$, we define:

- $S \subseteq S^{\prime} \stackrel{\text { def }}{\Longleftrightarrow} \forall \vec{x}: S(\vec{x}) \leq S^{\prime}(\vec{x})$

- $S \cup S^{\prime}:=\lambda \vec{x} \cdot S(\vec{x}) \vee S^{\prime}(\vec{x})$

- $S \cap S^{\prime}:=\lambda \vec{x} \cdot S(\vec{x}) \wedge S^{\prime}(\vec{x})$

We will now use the above fragment to first show how the archetypal alternativebased accounts of wh-questions and disjunction can be reproduced straightforwardly in possibility semantics; ${ }^{11}$ afterwards we will demonstrate that the compositionality issues discussed above no longer arise in this framework.

\subsubsection{Wh-questions}

As a first example, consider Hamblin's (1973) account of wh-questions, for which alternative semantics was originally developed. Hamblin assumes that who is of type $e$, but rather than denoting a single individual, it denotes the whole set of (human) individuals in the domain. By combining this denotation pointwise with, e.g., the meaning of sing, $\{\lambda x .|S x|\}$, Hamblin obtains the meaning of who sings:

$$
\llbracket \text { who sings } \rrbracket=\left\{|S x| \mid x \in D_{e}\right\}
$$

The same result may be obtained in possibility semantics without assuming that all expressions denote sets. However, who cannot be taken to have type $e$ in this setting, because that would mean that its semantic value is a specific individual. Instead, it has to be treated as being of type $\langle\langle e, T\rangle, T\rangle$, just like quantifiers:

$$
\llbracket \mathbf{w h o} \rrbracket=\lambda P_{\langle e, T\rangle} \cdot \bigcup_{x \in D_{e}} P x
$$

In words, the function denoted by who takes a function $P$ from individuals to sets of propositions, and returns the set consisting of all propositions which belong to the output of $P$ for some input individual $x$. It is easy to see that applying this function to the meaning of sing, or to anything of the same semantic type, results precisely in the meaning that Hamblin obtained by pointwise function application.

$$
\begin{aligned}
\llbracket \text { who sings } \rrbracket & =\llbracket \text { who } \rrbracket(\llbracket \text { sings } \rrbracket) \\
& =\left[\lambda P_{\langle e, T\rangle} \cdot \bigcup_{x \in D_{e}} P x\right](\lambda x \cdot\{|S x|\}) \\
& =\bigcup_{x \in D_{e}}\{|S x|\} \\
& =\left\{|S x| \mid x \in D_{e}\right\}
\end{aligned}
$$

\footnotetext{
11 Accounts of other linguistic constructions that have been formulated in alternative semantics are reproducible in possibility semantics as well, with one exception: the use of alternatives as a device for scope-taking (Shimoyama 2006), deriving exceptional scope phenomena as a consequence of pointwise function application. Since in possibility semantics the mode of composition is standard function application, such an account of scope-taking cannot be reproduced. We believe, however, that this is not a serious loss. In Appendix 1 we argue, building on Charlow (2014), that propagation of alternatives through pointwise function application does not provide a suitable basis for a general theory of exceptional scope. In order to obtain such a general theory within our framework, it would be possible to incorporate, e.g., ideas developed in Jäger (2007), Onea (2015) or Brasoveanu and Farkas (2011), where exceptional scope does not arise from alternative propagation. This enterprise, however, is beyond the scope of the present paper.
} 


\subsubsection{Disjunction}

In recent years, several analyses have been proposed that treat disjunction as an alternative-generating operation in order to account for, e.g., its semantic contribution in questions or when embedded under modals (von Stechow 1991; Simons 2005; Alonso-Ovalle 2006; Aloni 2007). By postulating that $\llbracket \boldsymbol{\alpha}$ or $\boldsymbol{\beta} \rrbracket=\llbracket \boldsymbol{\alpha} \rrbracket \cup \llbracket \boldsymbol{\beta} \rrbracket$, these accounts derive two separate alternatives for e.g. John sings or Mary dances, one for each disjunct, rather than just one disjunctive alternative.

$$
\begin{aligned}
\llbracket \text { John sings or Mary dances } \rrbracket & =\llbracket \text { John sings } \rrbracket \cup \llbracket \text { Mary dances } \rrbracket \\
& =\{|S j|\} \cup\{|D m|\} \\
& =\{|S j|,|D m|\}
\end{aligned}
$$

This may be reproduced categorematically in possibility semantics simply by associating sentential disjunction with its familiar meaning: $\llbracket$ or $_{T} \rrbracket=\lambda \mathcal{P}_{T} . \lambda \mathcal{Q}_{T} . \mathcal{P} \cup \mathcal{Q}$.

Since we dropped the assumption that all expressions denote sets, one may wonder how disjunctions of sub-sentential constituents can be handled in possibility semantics. To see this, consider the sentence John sings or dances. In alternative semantics, for the disjunctive VP we have:

$$
\begin{aligned}
\llbracket \text { sing or dance } \rrbracket & =\llbracket \text { sing } \rrbracket \cup \llbracket \text { dance } \rrbracket \\
& =\{\lambda x .|S x|\} \cup\{\lambda x .|D x|\} \\
& =\{\lambda x .|S x|, \lambda x .|D x|\}
\end{aligned}
$$

This set of properties then combines with $\llbracket \mathbf{J o h n} \rrbracket=\{j\}$ by means of pointwise function application, yielding $\{|S j|,|D j|\}$. Notice that the disjunctive verb phrase expresses a set of properties here. Thus, the alternatives that eventually emerge at the sentential level are already clearly visible at the verb phrase level.

In possibility semantics, the final result is the same, but it is obtained in a different way. We simply assume that disjunction is given its standard cross-categorical meaning (see, e.g., Partee and Rooth 1983):

$$
\begin{aligned}
& \text { For any conjoinable type } \tau \text { : } \\
& \begin{aligned}
\llbracket \mathbf{o r}_{\tau} \rrbracket & =\lambda X_{\tau} \cdot \lambda Y_{\tau} . X \cup Y \\
& =\lambda X_{\tau} \cdot \lambda Y_{\tau} . \lambda \vec{a} . X(\vec{a}) \vee Y(\vec{a})
\end{aligned}
\end{aligned}
$$

The verb phrase is then interpreted as follows. ${ }^{12}$

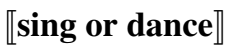

$$
\begin{aligned}
& =\llbracket \mathbf{o r}_{\langle e, T\rangle} \rrbracket(\llbracket \text { sing } \rrbracket)(\llbracket \text { dance } \rrbracket) \\
& =\left[\lambda P_{\langle e, T\rangle} \cdot \lambda Q_{\langle e, T\rangle} \cdot \lambda x_{e} \cdot \lambda p_{s t} . P(x)(p) \vee Q(x)(p)\right] \\
& \left(\lambda x_{e} \cdot \lambda p_{s t} \cdot p=|S x|\right)\left(\lambda x_{e} \cdot \lambda p_{s t} \cdot p=|D x|\right) \\
& =\lambda x \cdot \lambda p \cdot(p=|S x| \vee p=|D x|) \\
& =\lambda x \cdot\{|S x|,|D x|\}
\end{aligned}
$$

\footnotetext{
12 When deriving the semantic value of a disjunction $\llbracket \boldsymbol{\alpha}$ or $\boldsymbol{\beta} \rrbracket$, if the values of $\boldsymbol{\alpha}$ and $\boldsymbol{\beta}$ are construed as sets, the result can be directly computed by taking the standard union of these sets; it is easy to see that this coincides with the result given by our type-theoretic definition of union. However, when the values of $\boldsymbol{\alpha}$ and $\boldsymbol{\beta}$ are construed as functions, as in (13), it is crucial to rely on the explicit type-theoretic definition of the union operation.
} 
This function combines with $\llbracket \mathbf{J o h n} \rrbracket=j$ by means of standard function application, yielding the set $\{|S j|,|D j|\}$. Notice that in this case, the verb phrase does not express a set of properties, i.e., a set of functions from individuals to propositions, but rather a single function from individuals to sets of propositions. These sets of propositions only fully emerge at the sentential level. However, at the VP level they are already latently present, so to speak: the VP expresses an alternative-generating function, i.e., a function that, for any given input, produces a set of alternative propositions. Because of this shift in perspective, there is no need for pointwise function application.

\subsubsection{Standard function application regained}

Now let us verify that the compositionality issues that we discussed above for alternative semantics no longer arise in possibility semantics. The first of these issues had to do with pointwise function application, which makes it impossible for an operator to access the whole set of alternatives generated by its argument at once. Pointwise function application therefore necessitates a syncategorematic treatment of items like negation, disjunction, existential closure, and many others that need to operate on the whole set of alternatives generated by their argument. By contrast, since in possibility semantics meanings are composed by means of standard function application, there is nothing that prevents a categorematic treatment of these operators. After all, the input to the functor is the entire set of alternatives, rather than each alternative in isolation.

To illustrate this, consider sentential negation, which is now of type $\langle T, T\rangle$. That is, it expresses a function that takes a set of propositions into a new set of propositions. We obtain the desired result simply by defining $\llbracket \operatorname{not} \rrbracket=\lambda \mathcal{P}_{T} .\{\overline{\cup \mathcal{P}}\}$, and letting negation combine directly with its argument by standard function application. This is illustrated in the following derivation for John does not sing or dance.

a. $\llbracket$ John sings or dances $\rrbracket=\{|S j|,|D j|\}$

b. $\llbracket$ John does not sing or dance $\rrbracket=\llbracket$ not $\rrbracket(\{|S j|,|D j|\})$

$$
\begin{aligned}
& =\left[\lambda \mathcal{P}_{T} \cdot\{\overline{\cup \mathcal{P}}\}\right](\{|S j|,|D j|\}) \\
& =\{|S j| \cup|D j|\} \\
& =\left\{\left\{w \in D_{s}|w \notin| S j \mid \text { and } w \notin|D j|\right\}\right\}
\end{aligned}
$$

The result is a singleton set whose unique element is the proposition consisting of all worlds where John does not sing or dance, as desired.

\subsubsection{Standard predicate abstraction regained}

Turning to predicate abstraction, in possibility semantics there is no need to devise a special abstraction rule: the standard rule works fine. To see this in a simple example, consider the following syntactic tree for who did John see: 


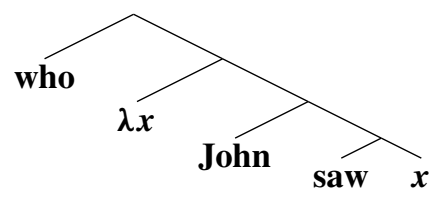

By function application we get that $\llbracket$ John saw $\boldsymbol{x} \rrbracket_{g}=\{|\operatorname{Sj} g(\boldsymbol{x})|\}$, a set containing a single proposition. Now, $\lambda \boldsymbol{x}$ is interpreted by means of the standard abstraction rule:

$$
\llbracket \lambda \boldsymbol{x} \text { John saw } \boldsymbol{x} \rrbracket_{g}=\lambda x_{e} \cdot \llbracket \text { John saw } \boldsymbol{x} \rrbracket_{g[x \mapsto x]}=\lambda x_{e} \cdot\{|\operatorname{Sj} x|\}
$$

The resulting constituent is of type $\langle e, T\rangle$, i.e., it expresses a function from individuals to sets of propositions. Applying the above entry for $\llbracket$ who $\rrbracket_{g}$ to this function yields the following set of propositions, as desired.

$$
\begin{aligned}
& \llbracket \text { who } \lambda \boldsymbol{x} \text { John saw } \boldsymbol{x} \rrbracket_{g}=\left[\lambda P_{\langle e, T\rangle} \cdot \bigcup_{x \in D_{e}} \operatorname{Px}\right]\left(\lambda x_{e} \cdot\{|S j x|\}\right) \\
& =\bigcup_{x \in D_{e}}\{|\operatorname{Sj} x|\} \\
& =\left\{|\operatorname{Sj} x| \mid x \in D_{e}\right\}
\end{aligned}
$$

Abstraction is unproblematic here, because it needs to deliver a single function from individuals to sets of propositions, rather than a set of functions from individuals to propositions.

Although we only gave a very small compositional fragment here, we hope it suffices to illustrate that theories which have been formulated in alternative semantics may generally be reproduced straightforwardly in possibility semantics. ${ }^{13}$ This allows us to handle the same phenomena in a mathematically more well-behaved setting, and frees us from the problems described above: first, since function application is no longer pointwise, operations that need access to the whole set of alternatives generated by their argument can be given a categorematic treatment; and second, we no longer need to look for a non-standard alternative-friendly abstraction rule. ${ }^{14}$

\section{Entailment}

Type theory does not only come with the operations of function application and abstraction which are used to compose meanings; it also comes with a notion of entailment which is used to compare meanings. This notion amounts to set-theoretic inclusion, and it applies cross-categorically to expressions of any conjoinable type. This general notion of entailment also gives rise to a principled cross-categorial treatment of conjunction and disjunction. Namely, if $\boldsymbol{\alpha}$ and $\boldsymbol{\beta}$ are expressions of any conjoinable type, then their conjunction $\alpha$ and $\boldsymbol{\beta}$ may be taken to denote the meet, i.e., the greatest lower

\footnotetext{
13 An exception to this claim is mentioned in footnote 11 and discussed in the Appendix.

14 The abstraction issue could also be circumvented by adopting a variable-free semantics. This, as briefly mentioned above, is the route that Shan (2004) takes. Such a semantics is based on combinatory logic rather than the lambda calculus, and does not involve abstraction at all. What we show here is that combining alternatives with the standard treatment of quantification does not necessitate a departure from the lambdacalculus.
} 
bound, of $\llbracket \boldsymbol{\alpha} \rrbracket$ and $\llbracket \boldsymbol{\beta} \rrbracket$ with respect to entailment. Dually, the disjunction $\boldsymbol{\alpha}$ or $\boldsymbol{\beta}$ may be taken to denote the join, i.e., the least upper bound, of $\llbracket \boldsymbol{\alpha} \rrbracket$ and $\llbracket \boldsymbol{\beta} \rrbracket$ with respect to entailment. ${ }^{15}$ It is easy to see that, for any two expressions $\boldsymbol{\alpha}$ and $\boldsymbol{\beta}$ of a conjoinable type, the meet of $\llbracket \boldsymbol{\alpha} \rrbracket$ and $\llbracket \boldsymbol{\beta} \rrbracket$ with respect to $\subseteq$ always exists, and amounts simply to the intersection $\llbracket \boldsymbol{\alpha} \rrbracket \cap \llbracket \boldsymbol{\beta} \rrbracket$; and similarly, the join of $\llbracket \boldsymbol{\alpha} \rrbracket$ and $\llbracket \boldsymbol{\beta} \rrbracket$ exists and amounts to the union $\llbracket \boldsymbol{\alpha} \rrbracket \cup \llbracket \boldsymbol{\beta} \rrbracket$.

Just like the composition rules of function application and abstraction, the crosscategorial treatment of entailment, as well as the cross-categorial treatment of conjunction and disjunction as meet and join operations that it gives rise to, are crucial features of the standard type-theoretic framework, which should not be lost in the process of moving to a more fine-grained notion of meaning.

Unfortunately, both in alternative semantics and in possibility semantics, the notion of entailment as set inclusion no longer gives sensible results. To see this, consider two basic sentences such as John walks and John moves: intuitively, the first sentence entails the second. In a classical semantic framework, this is captured by the typetheoretic notion of entailment: $\llbracket \mathbf{J o h n}$ walks $\rrbracket$ is the set $|W j|$ of worlds where John walks, and $\llbracket$ John moves $\rrbracket$ is the set $|M j|$ of worlds where John moves; since every world in which John walks is also a world in which John moves, we have $\left|W_{j}\right| \subseteq$ $|M j|$, and the entailment is predicted. However, in both alternative semantics and possibility semantics we have $\llbracket$ John walks $\rrbracket=\{|W j|\}$ and $\llbracket$ John moves $\rrbracket=\{|M j|\}$; since $\{|W j|\} \nsubseteq\{|M j|\}$, the entailment is not predicted. ${ }^{16}$

The general type-theoretic treatment of conjunction as intersection no longer gives desirable results in alternative/possibility semantics either. For instance, we would expect the conjunction John sings and Mary dances to express the singleton $\{\mid S j \wedge$ $D m \mid\}$, which has as its unique alternative the proposition that John sings and Mary dances. However, treating conjunction as intersection yields an absurd meaning:

$$
\llbracket \text { John sings and Mary dances } \rrbracket=\{|S j|\} \cap\{|D m|\}=\emptyset
$$

Just as for the compositionality problem, there are two ways to react to this problem: we may try to replace the standard type-theoretic notions of entailment and conjunction with pointwise counterparts which make suitable predictions in the alternative/possibility semantics framework; or, alternatively, we may reconsider some assumptions of our setup so that the standard type-theoretic notions may be recovered. We will first consider the first option, i.e., to define pointwise notions of entailment and conjunction. We will find, however, that such notions are problematic, and then turn to the second approach.

\footnotetext{
15 Formally, the meet of $a$ and $b$ with respect to a partial order $\leq$ is an element $c$ such that (i) $c \leq a, c \leq b$ and (ii) for any $d$ such that $d \leq a$ and $d \leq b$ it holds that $d \leq c$. Similarly for join. See, e.g., Keenan and Faltz (1985), Winter (2001), Roelofsen (2013) and Champollion (2016) for more background on these algebraic notions and their linguistic relevance.

16 This problem was first pointed out by Groenendijk and Stokhof (1984), who gave it as an argument against Hamblin's theory of questions. But as we have just seen, the problem in fact concerns alternative semantics more generally.
} 


\subsection{Entailment and conjunction in alternative/possibility semantics}

\subsubsection{Pointwise entailment}

Let us consider again the example illustrating the failure of standard entailment in alternative/possibility semantics: the problem is that the set of alternatives expressed by John walks is not a subset of the set of alternatives expressed by John moves; however, notice that the unique alternative for John walks is a subset of the unique alternative for John moves. This suggests that, instead of comparing the whole set of alternatives, in alternative/possibility semantics we should really be comparing the individual alternatives in the sets. More precisely, we may define entailment as pointwise inclusion: $\boldsymbol{\alpha}$ entails $\boldsymbol{\beta}$ in case every alternative for $\boldsymbol{\alpha}$ is included in some alternative for $\boldsymbol{\beta}$ :

$$
\boldsymbol{\alpha} \models \boldsymbol{\beta} \stackrel{\text { def }}{\Longleftrightarrow} \forall p \in \llbracket \boldsymbol{\alpha} \rrbracket \exists q \in \llbracket \boldsymbol{\beta} \rrbracket \text { such that } p \subseteq q
$$

This notion of entailment would indeed make the right predictions for basic cases: for instance, since the unique alternative for John walks, $|W j|$, is included in the unique alternative for John moves, $|M j|$, we would now correctly predict that John walks $\models$ John moves.

However, as discussed in Roelofsen (2013), there is a fundamental problem with this notion. Namely, entailment defined in this way does not amount to a partial order on the space of meanings. In particular, it is not anti-symmetric, which means that two expressions $\boldsymbol{\alpha}$ and $\boldsymbol{\beta}$ may be logically equivalent — that is, entail each other-and yet have different meanings. To see this, consider the following two sentences ${ }^{17}$ :

$$
\begin{aligned}
& \llbracket \text { John moves } \rrbracket=\{|M j|\} \\
& \llbracket \text { John moves or walks } \rrbracket=\{|M j|,|W j|\}
\end{aligned}
$$

Since the proposition $\left|W_{j}\right|$ that John walks is contained in the proposition $|M j|$ that John moves, every alternative for John moves or walks is contained in an alternative for John moves. Vice versa, the unique alternative for John moves is clearly contained in one of the alternatives for John moves or walks. Thus, the two sentences entail each other, but they have different meanings. ${ }^{18}$

In a classical intensional framework, if two sentences are logically equivalent, this implies that they are synonymous, i.e., they have the same meaning. We would like the notion of logical equivalence in our framework to behave in this classical

\footnotetext{
17 For concreteness, we assume in our examples that disjunction has the alternative-generating behavior argued for by Simons (2005), Alonso-Ovalle (2006), and Aloni (2007). Nothing hinges on this assumption, though, and the reader should feel free to replace disjunction with her favorite alternative-generating item.

18 Notice that in classical, truth-conditional semantics, the two sentences have the same meaning. This fact has been used to explain the oddness of disjunctions like John moves or walks in terms of redundancy; after all, in a truth-conditional semantics the second disjunct does not make any contribution to the disjunction as a whole (Katzir and Singh 2013). If we want to preserve this explanation, the two sentences also have to be assigned the same meaning in an alternative-based semantics. We will see in Sect. 3.3.2 that this is indeed achieved in the system we propose.
} 
way as well. This means that, if a certain equivalence relation doesn't guarantee that equivalence implies synonymy, then it is not a suitable notion of logical equivalence for the framework at hand. Hence, the relation $\models$ defined above does not qualify as a satisfactory notion of entailment in alternative semantics.

This conceptual problem has practical repercussions as well. For instance, if entailment is not a partial order on the space of meanings, conjunction and disjunction can no longer be treated as meet and join operations with respect to entailment. Consider conjunction: we would like to define $\llbracket \boldsymbol{\alpha}$ and $\boldsymbol{\beta} \rrbracket$ as the meet of $\llbracket \boldsymbol{\alpha} \rrbracket$ and $\llbracket \boldsymbol{\beta} \rrbracket$, i.e., as the weakest meaning entailing both $\llbracket \boldsymbol{\alpha} \rrbracket$ and $\llbracket \boldsymbol{\beta} \rrbracket$. However, since pointwise entailment is not anti-symmetric, there is not a unique such meaning, but rather a whole cluster of them, and we have no principled way to single out one particular element from this cluster. This means that we lose our principled account of conjunction and disjunction in terms of cross-categorial meet and join operations. Thus, redefining entailment as pointwise inclusion is unsatisfactory.

\subsubsection{Pointwise conjunction}

Setting the general problem with entailment aside, we may still try to devise an alternative-friendly notion of conjunction that avoids the problematic predictions resulting from treating conjunction as intersection. Recall our example: we have $\llbracket$ John sings $\rrbracket=\{|S j|\}, \llbracket$ Mary dances $\rrbracket=\{|D m|\}$, and we want $\llbracket$ John sings and Mary dances $\rrbracket=\{|S j \wedge D m|\}=\{|S j| \cap|D m|\}$. This suggests that, rather than intersecting two meanings directly, conjunction should be intersecting the individual alternatives within these meanings. More precisely, it suggests the following treatment of conjunction as pointwise intersection:

$$
\llbracket \text { and } \rrbracket=\lambda P . \lambda Q \cdot\{p \cap q \mid p \in P \text { and } q \in Q\}
$$

Again, for the most basic cases, this treatment makes the right predictions. For instance, we do indeed get that $\llbracket$ John sings and Mary dances $\rrbracket=\{|S j| \cap|D m|\}=$ $\{|S j \wedge D m|\}$; and this extends more generally to all cases where both conjuncts have singleton meanings. However, with non-singleton conjuncts, pointwise intersection often yields spurious alternatives. For instance, we expect that conjoining a sentence with itself will make no difference to its meaning, i.e., we expect conjunction to be idempotent: for any sentence $\boldsymbol{\alpha}, \llbracket \boldsymbol{\alpha} \wedge \boldsymbol{\alpha} \rrbracket=\llbracket \boldsymbol{\alpha} \rrbracket$. But that is not generally the case. Consider a sentence with two alternatives, such as $\boldsymbol{\alpha}=$ John sang or danced. Besides the two expected alternatives $|S j|$ and $|D j|$, the conjunction $\boldsymbol{\alpha} \wedge \boldsymbol{\alpha}$ also generates a third alternative, namely the proposition $|S j \wedge D j|$ that John sang and danced.

$$
\llbracket \text { John sang or danced and John sang or danced } \rrbracket=\{|S j|,|D j|,|S j \wedge D j|\}
$$

We see no reason why conjunction should give rise to this extra alternative, and we doubt that empirical support for this prediction may be found. A similar issue also arises for other items whose meaning relies on set intersection, such as universal 
quantification: if we take a universal quantifier to perform pointwise intersection, even a vacuous universal quantifier may introduce spurious alternatives. ${ }^{19}$

\subsection{Entailment and conjunction in inquisitive semantics}

\subsubsection{Recovering standard entailment and conjunction}

Given that adapting the notions of entailment and conjunction to alternative/possibility semantics is not a trivial affair, it is worth considering once more the strategy we adopted in Sect. 2 to deal with the compositionality problem: identify exactly which features of the framework are responsible for the problem, and ask whether it is possible to modify these features so that the problem is avoided, while the desirable features of the framework are retained.

In order to do this, let us look once more at the example illustrating the problem

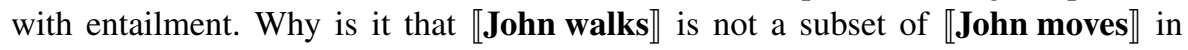
alternative/possibility semantics? This is because both meanings are singleton sets, consisting of the unique alternative for the sentence. The assumption that a basic sentence $\boldsymbol{\alpha}$ denotes the singleton $\{|\boldsymbol{\alpha}|\}$, shared by alternative and possibility semantics, may seem quite innocent: after all, the standard meaning of a sentence $\alpha$ is a single proposition, $|\boldsymbol{\alpha}|$, and if we want to represent this meaning as a set of propositions, what better candidate than the singleton set containing just $|\boldsymbol{\alpha}|$ ? However, the problems with entailment and conjunction indicate that identifying classical propositions with the corresponding singleton sets may not be the best way of embedding classical semantics into alternative semantics after all.

It is certainly natural to regard a basic sentence like John walks as introducing a unique alternative, namely, the proposition $|W j|$. But it does not follow from this that we have to construe the meaning of John walks as the singleton set $\{|W j|\}$. To enjoy the benefits of having alternatives in our semantics, it is not necessary to assume that the meaning of a sentence is identical with the set of alternatives that the sentence introduces; it is sufficient to assume that the meaning of a sentence determines the set of alternatives that it introduces.

What, then, should we take to be the meaning of a basic sentence like John walks? Let us examine carefully what the desiderata are. Suppose $\boldsymbol{\alpha}$ and $\boldsymbol{\beta}$ are two basic sentences, that is, two sentences having as their unique alternative the proposition that they classically express. For such sentences, we want the standard, truth-conditional notion of entailment to be preserved. That is, $\boldsymbol{\alpha}=\boldsymbol{\beta}$ should hold just in case $|\boldsymbol{\alpha}| \subseteq|\boldsymbol{\beta}|$. Moreover, we want to preserve the standard type-theoretic conception of entailment

\footnotetext{
19 A reviewer suggests that it may be possible to resolve the problem by defining conjunction in such a way that it returns only the maximal propositions that result from pointwise intersecting the proposition sets associated with the two conjuncts. This would indeed give the desired result for the example discussed here, but it would not solve the more general problem, i.e., it would not make conjunction idempotent. For instance, if $\boldsymbol{\alpha}=$ John walks or moves, then $\llbracket \alpha \rrbracket=\{|W j|,|M j|\}$, where $|W j| \subseteq|M j|$. Under the suggested treatment of conjunction we would get that $\llbracket \alpha \wedge \alpha \rrbracket=\{|M j|\} \neq \llbracket \alpha \rrbracket$. Here, too, we see no reason why $\alpha \wedge \boldsymbol{\alpha}$ should not just be associated with the same alternative set as $\boldsymbol{\alpha}$ itself.
} 
as meaning inclusion, so $\boldsymbol{\alpha} \models \boldsymbol{\beta}$ should amount to $\llbracket \boldsymbol{\alpha} \rrbracket \subseteq \llbracket \boldsymbol{\beta} \rrbracket$. To satisfy these two desiderata, we need to make sure that $\llbracket \boldsymbol{\alpha} \rrbracket$ and $\llbracket \boldsymbol{\beta} \rrbracket$ are construed in such a way that:

$$
|\boldsymbol{\alpha}| \subseteq|\boldsymbol{\beta}| \Longleftrightarrow \llbracket \boldsymbol{\alpha} \rrbracket \subseteq \llbracket \boldsymbol{\beta} \rrbracket
$$

This result is naturally obtained if we do not construe $\llbracket \boldsymbol{\alpha} \rrbracket$ and $\llbracket \boldsymbol{\beta} \rrbracket$ as the singleton sets $\{|\boldsymbol{\alpha}|\}$ and $\{|\boldsymbol{\beta}|\}$, respectively, but rather as the powersets $\wp(|\boldsymbol{\alpha}|)$ and $\wp(|\boldsymbol{\beta}|)$, i.e., the set of all subsets of $|\boldsymbol{\alpha}|$ and $|\boldsymbol{\beta}|$, respectively. Clearly, if $|\boldsymbol{\alpha}| \subseteq|\boldsymbol{\beta}|$, then any subset of $|\boldsymbol{\alpha}|$ is also a subset of $|\boldsymbol{\beta}|$. And conversely, if any subset of $|\boldsymbol{\alpha}|$ is a subset of $|\boldsymbol{\beta}|$, then it follows that $|\boldsymbol{\alpha}| \subseteq|\boldsymbol{\beta}|$. Intuitively, we take the meaning of John walks to be the set of all propositions that contain enough information to establish that John walks, i.e., all propositions $p$ such that John walks in every world in $p$, rather than just the proposition that contains precisely the information that John walks, i.e., the proposition consisting of all worlds in which John walks.

This does not mean that we give up the idea that John walks has a unique alternative: for, we can recover the unique alternative for John walks as the maximal element of its meaning. This is precisely the set of all worlds where John walks. Thus, by distinguishing the meaning of a sentence from the alternatives it introduces, we can simultaneously retain the usual alternatives for the sentence on the one hand, and the standard type-theoretic notion of entailment on the other.

The reasoning just outlined for basic sentences with a single alternative can be generalized to sentences with multiple alternatives as well. In the spirit of Hamblin (1973) as well as inquisitive semantics (see, e.g., Ciardelli et al. 2013, 2015) such sentences can be thought of as raising an issue as to which of the alternatives contains the actual world. Crucially, while Hamblin originally identified the meaning of a sentence with the alternatives it introduces, inquisitive semantics dissociates the two notions in precisely the way discussed above for basic sentences. That is, the meaning of a sentence in inquisitive semantics consists of all propositions that contain enough information to resolve the issue that the sentence raises, rather than just those that contain precisely the information that is needed to do so. As a consequence, sentential meanings in inquisitive semantics are not unconstrained sets of propositions, as in alternative/possibility semantics, but rather sets of propositions that are downward closed: if $\llbracket \boldsymbol{\alpha} \rrbracket$ includes a proposition $p$ then it also includes every stronger proposition $q \subseteq p$. After all, if $p$ contains enough information to resolve the issue that $\alpha$ raises, then any $q \subseteq p$ will also contain enough information to do so.

We will refer to downward closed sets of propositions as inquisitive meanings and to the result of making a set of propositions $\mathcal{P}$ downward closed as the downward closure of that set, written as $\mathcal{P}^{\downarrow}$.

$$
\mathcal{P} \downarrow \stackrel{\text { def }}{=}\{q \mid q \subseteq p \text { for some } p \in \mathcal{P}\}
$$

Given the inquisitive meaning $\llbracket \boldsymbol{\alpha} \rrbracket$ of a sentence $\boldsymbol{\alpha}$, the alternatives that $\boldsymbol{\alpha}$ generates can be identified with the maximal elements of $\llbracket \alpha \rrbracket$. Intuitively, these propositions contain 
sufficient information to resolve the issue raised by $\boldsymbol{\alpha}$, and not more information than necessary to do so. ${ }^{20}$

$$
\operatorname{ALT}(\boldsymbol{\alpha}) \stackrel{\text { def }}{=}\{p \in \llbracket \boldsymbol{\alpha} \rrbracket \mid \text { there is no } q \in \llbracket \boldsymbol{\alpha} \rrbracket \text { such that } p \subset q\}
$$

Crucially, while the inquisitive perspective on meaning allows us to associate a set of alternatives with every sentence, it also allows us to recover the standard type-theoretic notion of entailment as inclusion. This notion of entailment constitutes a well-behaved partial order on the space of inquisitive meanings. In particular, it is anti-symmetric, which means that any two expressions that are logically equivalent express the same meaning, as desired.

Furthermore, as shown in Roelofsen (2013), the space of inquisitive meanings ordered by entailment forms a complete Heyting algebra, just like the space of classical propositions ordered by classical entailment. This means in particular that two inquisitive meanings $\mathcal{P}$ and $\mathcal{Q}$ always have:

- a meet, i.e., a unique greatest lower bound w.r.t. entailment, given by $\mathcal{P} \cap \mathcal{Q}$

- a join, i.e., a unique least upper bound w.r.t. entailment, given by $\mathcal{P} \cup \mathcal{Q}$

As a consequence, we can restore the standard treatment of conjunction and disjunction as meet and join operations. Moreover, these operations still amount to intersection and union, just as in the classical type-theoretic framework.

$$
\begin{aligned}
& \llbracket \text { and } \rrbracket=\lambda \mathcal{P} . \lambda \mathcal{Q} . \mathcal{P} \cap \mathcal{Q} \\
& \llbracket \text { or } \rrbracket=\lambda \mathcal{P} . \lambda \mathcal{Q} . \mathcal{P} \cup \mathcal{Q}
\end{aligned}
$$

We will see in a moment that these entries for sentential conjunction and disjunction can be generalized to entries that admit conjuncts/disjuncts of any conjoinable type.

\subsubsection{Negation and universal quantification}

The fact that inquisitive meanings form a complete Heyting algebra ensures that, besides the meet and join operations, there are two other general algebraic operations that can be performed on inquisitive meanings as well, which will allow us to restore the standard treatment of negation and universal quantification, respectively.

The first of these operations is pseudo-complementation. The pseudo-complement of an inquisitive meaning $\mathcal{P}$ is the weakest inquisitive meaning $\mathcal{Q}$ whose meet with $\mathcal{P}$ is inconsistent, i.e., such that $\mathcal{P} \cap \mathcal{Q}=\{\emptyset\}$. The fact that inquisitive meanings form a Heyting algebra guarantees that such a pseudo-complement always exists. We will denote it as $\rightarrow \mathcal{P}$, and will refer to the operation $\rightarrow$ as inquisitive negation. There is a simple recipe to compute $\rightarrow \mathcal{P}$ for any given inquisitive meaning $\mathcal{P}$. Namely, $\rightarrow \mathcal{P}$ amounts to the set of propositions that are incompatible with every element of $\mathcal{P}$.

$$
\neg \mathcal{P}=\{p \mid p \cap q=\emptyset \text { for all } q \in \mathcal{P}\}
$$

\footnotetext{
20 This approach imposes a constraint on the kinds of alternative sets that may be associated with a sentence. Namely, if $p$ and $q$ are two alternatives associated with a sentence $\boldsymbol{\alpha}$, we must have that $p \not \subset q$ and $q \not \subset p$, neither one can be nested in the other. This has interesting repercussions for the analysis of so-called Hurford disjunctions, like John moves or walks. We will briefly return to this point in footnote 23 .
} 
Just like in classical type-theoretic semantics we may now treat sentential negation as expressing pseudo-complementation; and again, we will see in a moment that this treatment can be generalized straightforwardly so as to apply to non-sentential constituents as well.

$$
\llbracket \operatorname{not} \rrbracket=\lambda \mathcal{P} . \rightarrow \mathcal{P}
$$

An observation that will be useful in dealing with some of the examples to be considered below is that $\rightarrow \mathcal{P}$ always has a unique maximal element, i.e., a unique alternative, namely $\overline{\cup \mathcal{P}}$. Thus, $\rightarrow \mathcal{P}$ may be characterized as follows:

$$
\neg \mathcal{P}=\{\overline{\bigcup \mathcal{P}}\}^{\downarrow}
$$

Now let us turn to the other algebraic operation, which is called relative pseudocomplementation. The pseudo-complement of an inquisitive meaning $\mathcal{P}$ relative to another inquisitive meaning $\mathcal{Q}$ is the weakest inquisitive meaning $\mathcal{R}$ whose meet with $\mathcal{P}$ entails $\mathcal{Q}$, i.e., such that $\mathcal{P} \cap \mathcal{R} \models \mathcal{Q}$. The fact that inquisitive meanings form a Heyting algebra again guarantees that such a relative pseudo-complement always exists. We will denote it as $\mathcal{P} \rightarrow \mathcal{Q}$, and will refer to the operation $\rightarrow$ as inquisitive implication. Just as in the case of $\rightarrow \mathcal{P}$, there is a simple recipe that can be used to compute $\mathcal{P} \rightarrow \mathcal{Q}$ for any two inquisitive meanings $\mathcal{P}$ and $\mathcal{Q}$. Namely, $\mathcal{P} \rightarrow \mathcal{Q}$ amounts to the set of propositions $p$ such that for every $p^{\prime} \subseteq p$, if $p^{\prime} \in \mathcal{P}$ then $p^{\prime} \in \mathcal{Q}$ as well. ${ }^{21}$

$$
\mathcal{P} \rightarrow \mathcal{Q}=\left\{p \mid \text { for every } p^{\prime} \subseteq p: \text { if } p^{\prime} \in \mathcal{P} \text { then } p^{\prime} \in \mathcal{Q}\right\}
$$

Relative pseudo-complementation plays a crucial role in the classical treatment of the determiner every. In alternative/possibility semantics this treatment is lost, because the underlying algebraic structure of the classical framework is not maintained. In inquisitive semantics, on the other hand, it is naturally recovered:

$$
\llbracket \text { every } \rrbracket=\lambda P_{\langle e, T\rangle} \lambda Q_{\langle e, T\rangle} \cdot \bigcap_{x \in D}(P x \rightarrow Q x)
$$

In a moment, we will see several examples illustrating the consequences of this treatment of every.

\subsection{A compositional inquisitive semantics fragment}

We now specify a compositional inquisitive semantics for a small fragment of English, extending the possibility semantics fragment in Sect. 2.2.

\footnotetext{
${ }^{21}$ It may be useful to note that, since the set of all propositions $p^{\prime}$ such that $p^{\prime} \subseteq p$ and $p^{\prime} \in \mathcal{P}$ can be denoted compactly as $\wp(p) \cap \mathcal{P}$, we could also characterize $\mathcal{P} \rightarrow \mathcal{Q}$ as follows:
}

(i) $\quad \mathcal{P} \rightarrow \mathcal{Q}=\{p \mid \wp(p) \cap \mathcal{P} \subseteq \mathcal{Q}\}$ 
- Proper names and variables:

$$
\begin{aligned}
& \text { - } \llbracket \mathbf{J o h n} \rrbracket_{g}=j \\
& \text { - } \llbracket \boldsymbol{x} \rrbracket_{g}=g(\boldsymbol{x})
\end{aligned}
$$

- Connectives:

$$
\begin{aligned}
& \text { - } \llbracket \mathbf{o r}_{\tau} \rrbracket=\lambda X_{\tau} \lambda Y_{\tau} . X \cup Y \\
& \text { - } \llbracket \operatorname{and}_{\tau} \rrbracket=\lambda X_{\tau} \lambda Y_{\tau} . X \cap Y \\
& \text { - } \llbracket \operatorname{not}_{T} \rrbracket=\lambda \mathcal{P}_{T} \cdot \neg \mathcal{P} \\
& \text { - } \llbracket \operatorname{not}_{\langle e, T\rangle} \rrbracket=\lambda P_{\langle e, T\rangle} \cdot \lambda x . \sqcap P x
\end{aligned}
$$

- Existential closure:

$$
\text { - } \llbracket \exists \rrbracket=\lambda \mathcal{P}_{T} \cdot\{\bigcup \mathcal{P}\}^{\downarrow}
$$

We will comment on the various elements of this fragment, focusing on differences w.r.t. alternative/possibility semantics.

\subsubsection{Verbs, nouns, proper names, and variables}

In inquisitive semantics we want the meaning of a simple sentence like John walks to be a downward closed set of propositions, namely $\{|W j|\}^{\downarrow}$. So we let the verb walk denote a function that maps any individual $x$ to the set of propositions which contain enough information to establish that $x$ walks.

$$
\begin{aligned}
\llbracket \mathbf{w a l k s} \rrbracket & =\lambda x .\{|W x|\}^{\downarrow} \\
& =\lambda x .\{p \mid x \text { walks in every } w \in p\}
\end{aligned}
$$

A proper name like John just denotes an individual (rather than the singleton set containing that individual, as in alternative semantics), and the same goes for variables. If such an individual combines with the denotation of a verb like walk, the resulting meaning will be a downward closed set of propositions:

$$
\begin{aligned}
\llbracket \mathbf{w a l k s} \rrbracket(\llbracket \mathbf{J o h n} \rrbracket) & =\left[\lambda x .\{|W x|\}^{\downarrow}\right](j) \\
& =\{|W j|\}^{\downarrow} \\
& =\{p \mid \text { John walks in every } w \in p\}
\end{aligned}
$$

\subsubsection{Connectives}

We already saw above that the inquisitive notion of meaning allows us to restore the standard treatment of sentential conjunction and disjunction as meet and join operations. This result generalizes to arbitrary conjoinable types, yielding a crosscategorical account of conjunction and disjunction. For instance, for the $\langle e, T\rangle$-type disjunction sing or dance we get: ${ }^{22}$

\footnotetext{
22 Recall that we use set-theoretic notation as an abbreviation for type-theoretic notation. Thus, for instance, our entry for sing, $\lambda x .\{|S x|\}^{\downarrow}$, is to be regarded as an abbreviation for $\lambda x . \lambda p .(p \subseteq|S x|)$. Also, recall that we work with the explicit type-theoretic definition of union whenever the arguments are regarded as functions rather than sets, as in (35).
} 


$$
\begin{aligned}
& \llbracket \text { sing or dance } \rrbracket \\
& =\llbracket \text { or } \rrbracket(\llbracket \text { sing } \rrbracket)(\llbracket \text { dance } \rrbracket) \\
& =\left[\lambda P_{\langle e, T\rangle} \cdot \lambda Q_{\langle e, T\rangle} \cdot \lambda x_{e} \cdot \lambda p_{s t} . P(x)(p) \vee Q(x)(p)\right]\left(\lambda x_{e} \cdot \lambda p_{s t} \cdot p \subseteq|S x|\right) \\
& \quad\left(\lambda x_{e} \cdot \lambda p_{s t} \cdot p \subseteq|D x|\right) \\
& =\lambda x_{e} \cdot \lambda p_{s t} \cdot(p \subseteq|S x| \vee p \subseteq|D x|) \\
& =\lambda x_{e} \cdot\{|S x|,|D x|\}^{\downarrow}
\end{aligned}
$$

As in alternative semantics, disjunction typically generates alternatives. For instance:

$$
\begin{aligned}
\llbracket \text { John sings or Mary dances } \rrbracket & =\llbracket \text { John sings } \rrbracket \cup \llbracket \text { Mary dances } \rrbracket \\
& =\{|S j|,|D m|\}^{\downarrow}
\end{aligned}
$$

This meaning has two maximal elements, namely, the proposition that John sings, and the proposition that Mary dances:

$$
\operatorname{ALT}(\text { John sings or Mary dances })=\{|S j|,|D m|\}
$$

Thus, we recover the alternative-generating treatment of disjunction that was argued for on an empirical basis by Simons (2005), Alonso-Ovalle (2006) and Aloni (2007). However, now this behavior is not stipulated, but follows from the standard treatment of disjunction as a join operation in the given space of meanings (cf. Roelofsen 2015). ${ }^{23}$

Next, consider conjunction. Restoring the standard treatment of conjunction as a meet operator does not only re-establish the link between entailment and conjunction, but also resolves the empirical problems pointed out above. First, performing intersection now yields the right results for cases that were problematic in alternative and possibility semantics.

$$
\begin{aligned}
\llbracket \text { John sings and Mary dances } \rrbracket & =\llbracket \text { John sings } \rrbracket \cap \llbracket \text { Mary dances } \rrbracket \\
& =\{|S j|\}^{\downarrow} \cap\{|D m|\}^{\downarrow} \\
& =\{|S j| \cap|D m|\}^{\downarrow} \\
& =\{|S j \wedge D m|\}^{\downarrow}
\end{aligned}
$$

As desired, John sings and Mary dances is predicted to have a unique alternative, namely, the proposition that John sings and Mary dances. Moreover, unlike the pointwise conjunction operation that we considered above, intersection is obviously idempotent, which means that the problem with spurious alternatives no longer arises:

$$
\operatorname{ALT}(\text { John sings or dances and John sings or dances })=\{|S j|,|D j|\}
$$

More generally, since conjunction is treated again as performing the meet operation with respect to entailment, it regains its familiar, well-understood logical features.

\footnotetext{
23 Note that if one disjunct entails the other, as in John moves or walks, then the resulting meaning contains just one alternative, namely $|M j|$. This makes it possible to explain the oddness of these so-called Hurford disjunctions (cf. Hurford 1974; Chierchia et al. 2009; Katzir and Singh 2013) in terms of redundancy. After all, John moves or walks is synonymous with just John moves; the second disjunct does not make any contribution to the meaning of the disjunction as a whole. On the other hand, in alternative semantics the meaning of John moves or walks consists of two alternatives, $|M j|$ and $|W j|$, one of which is contained in the other (as we saw in Sect. 3.1.1). Thus, in alternative semantics John moves or walks is not synonymous with just John moves, which means that its oddness cannot be explained directly in terms of redundancy (this point is discussed in more detail in Ciardelli and Roelofsen 2016).
} 
The problems discussed above for pointwise conjunction do not only concern connectives like and. Rather, all items with a conjunctive semantics are affected, including universal quantifiers like everybody and determiners like which. These items, which we will consider in more detail momentarily, can now be treated in terms of plain intersection. ${ }^{24}$

Let us now turn to negation. We have seen above that the inquisitive notion of meaning enables us to capture sentential negation as pseudo-complementation, as in the classical type-theoretic framework. This result can be generalized in order to obtain a treatment of negation that applies to constituents of type $\langle e, T\rangle$, such as verb phrases.

$$
\llbracket \operatorname{not}_{\langle e, T\rangle} \rrbracket=\lambda P_{\langle e, T\rangle} \cdot \lambda x . \neg P x
$$

For instance, applying this generalized negation to dance of type $\langle e, T\rangle$, we again obtain an item of type $\langle e, T\rangle$. As desired, not dance denotes a function that maps an individual to a set of propositions consisting exclusively of worlds in which the individual doesn't dance.

$$
\begin{aligned}
& \llbracket \text { not dance } \rrbracket=\llbracket \text { not } \rrbracket(\llbracket \text { dance } \rrbracket) \\
& =\left[\lambda P_{\langle e, T\rangle} \cdot \lambda x . \rightarrow P x\right]\left(\lambda x .\{|D x|\}^{\downarrow}\right) \\
& =\lambda x . \rightarrow\{|D x|\}^{\downarrow} \\
& =\lambda x .\{p|w \notin| D x \mid \text { for all } w \in p\} \\
& =\lambda x \cdot\{|\neg D x|\}^{\downarrow}
\end{aligned}
$$

Note that negation, both at sentence and at VP level, has the effect of flattening its argument set, meaning that the set of propositions arising from a negated constituent always contains a unique alternative. While this effect doesn't make a difference in the example above since dance only generates a single alternative to begin with, it is crucial in the following derivation, where negation applies to a disjunctive verb phrase.

$$
\begin{aligned}
\llbracket \text { not sing or dance } \rrbracket & =\llbracket \text { not } \rrbracket(\llbracket \text { sing } \rrbracket \cup \llbracket \text { dance } \rrbracket) \\
& =[\lambda P\langle e, T\rangle \cdot \lambda x . \neg P x]\left(\lambda x .\left(\{|S x|\}^{\downarrow} \cup\{|D x|\}^{\downarrow}\right)\right) \\
& =\lambda x . \neg\left(\{|S x|\}^{\downarrow} \cup\{|D x|\}^{\downarrow}\right) \\
& =\lambda x .\{p|w \notin| S x \mid \text { and } w \notin|D x| \text { for all } w \in p\} \\
& =\lambda x .\{|\neg S x \wedge \neg D x|\}^{\downarrow}
\end{aligned}
$$

As expected, not sing or dance denotes a function that maps every individual $x$ to the set of propositions consisting only of worlds where $x$ neither sings nor dances. This set of propositions contains a unique alternative, namely the set of all worlds where $x$ neither sings nor dances.

\subsubsection{Quantifiers}

Since the inquisitive notion of meaning enriches the classical one, the denotations of generalized quantifiers (GQs) in our inquisitive semantics fragment necessarily differ

\footnotetext{
${ }^{24} \mathrm{Had}$ we wanted to include entries for these items in the possibility semantics fragment from Section 2 , we would have had to rely on pointwise intersection.
} 
from those in a classical extensional framework. They do, however, closely mirror classical GQs. Let us see how exactly.

In an extensional setting, the denotation of a sentence in a world is a truth value. This is reflected in the semantic objects assigned to GQs. These semantic objects, which we will also just call GQs, are of type $\langle\langle e, t\rangle, t\rangle$, i.e., sets of properties. A GQ like every man can thus combine with a verb phrase of type $\langle e, t\rangle$, such as sings; the end result is of type $t$. One important feature of this setup is that GQs, being sets of properties, can be compounded by means of algebraic operations like meet and join. For instance, the GQ expressed by every man and no woman can be obtained by taking the intersection (meet) of the GQ expressed by every man and that expressed by no woman.

In inquisitive semantics, the meaning of a sentence is a set of propositions. In the same way as in the classical setting, this is reflected in the semantic objects assigned to GQs, which now have type $\langle\langle e, T\rangle, T\rangle$. A GQ like every man can still combine with a verb phrase like sings. The difference is that the latter is now of type $\langle e, T\rangle$ and the end result is of type $T$.

$$
\begin{aligned}
\llbracket \text { every man sings } \rrbracket & =\llbracket \text { every man } \rrbracket(\llbracket \text { sings } \rrbracket) \\
& =\left[\lambda P_{\langle e, T\rangle} \bigcap_{x \in D_{e}}\left(\{|M x|\}^{\downarrow} \rightarrow P x\right)\right]\left(\lambda x .\{|S x|\}^{\downarrow}\right) \\
& =\bigcap_{x \in D_{e}}\left(\{|M x|\}^{\downarrow} \rightarrow\{|S x|\}^{\downarrow}\right) \\
& =\bigcap_{x \in D_{e}}\{p|p \cap| M x|\subseteq| S x \mid\} \\
& =\left\{p\left|\forall x \in D_{e}: p \cap\right| M x|\subseteq| S x \mid\right\}
\end{aligned}
$$

The resulting set of propositions contains one maximal element, which is the set of worlds $w$ where the set of men, $M_{w}$, is a subset of the set of individuals who sing, $S_{w}$. So the meaning of every man sings amounts to $\left\{\left\{w \mid M_{w} \subseteq S_{w}\right\}\right\}^{\downarrow}$.

Even though GQs are now more complex, they still have many of the attractive properties of classical GQs. For instance, just as before, they can be coordinated by means of generalized disjunction and conjunction. To see how, consider the following GQs from our inquisitive semantics fragment.

$$
\begin{aligned}
& \text { a. } \quad \text { every man } \rrbracket=\lambda P_{\langle e, T\rangle} \cdot \bigcap_{x \in D_{e}}\left(\{|M x|\}^{\downarrow} \rightarrow P x\right) \\
& \text { b. } \quad \llbracket \text { no woman } \rrbracket=\lambda P_{\langle e, T\rangle} \cdot \bigcap_{x \in D_{e}}\left(\{|W x|\}^{\downarrow} \rightarrow \sim P x\right)
\end{aligned}
$$

These lambda terms denote functions that map 'inquisitive properties' (functions from individuals to inquisitive meanings) to sets of propositions. Like any objects of a conjoinable type, these functions can be regarded as encoding sets of $n$-tuples, i.e., relations, in this case between 'inquisitive properties' and propositions.

$$
\begin{aligned}
& \text { a. } \quad \llbracket \text { every man } \rrbracket=\left\{\left\langle P_{\langle e, T\rangle}, p_{\langle s, t\rangle}\right\rangle \mid p \in \bigcap_{x \in D_{e}}\left(\{|M x|\}^{\downarrow} \rightarrow P x\right)\right\} \\
& \text { b. } \quad \llbracket \text { no woman } \rrbracket=\left\{\left\langle P_{\langle e, T\rangle}, p_{\langle s, t\rangle}\right\rangle \mid p \in \bigcap_{x \in D_{e}}\left(\{|W x|\}^{\downarrow} \rightarrow \rightarrow P x\right)\right\}
\end{aligned}
$$

From this set representation, it is clear that we can take, just like in the classical setting, the intersection and union of these GQs. For instance, we can now obtain the 
GQ expressed by every man and no woman by taking the intersection (meet) of the above sets:

$$
\begin{aligned}
& \llbracket \text { every man and no woman } \rrbracket \\
& \quad=\llbracket \text { every man } \rrbracket \cap \llbracket \text { no woman } \rrbracket \\
& \quad=\left\{\left\langle P_{\langle e, T\rangle}, p_{\langle s, t\rangle}\right\rangle \mid p \in \bigcap_{x \in D_{e}}\left(\{|M x|\}^{\downarrow} \rightarrow P x\right) \text { and } p \in \bigcap_{x \in D_{e}}\left(\{|W x|\}^{\downarrow} \rightarrow \rightarrow P x\right)\right\} \\
& \quad=\left\{\left\langle P_{\langle e, T\rangle}, p_{\langle s, t\rangle}\right\rangle \mid p \in \bigcap_{x \in D_{e}}\left(\left(\{|M x|\}^{\downarrow} \rightarrow P x\right) \cap\left(\{|W x|\}^{\downarrow} \rightarrow \rightarrow P x\right)\right)\right\} \\
& \quad=\lambda P_{\langle e, T\rangle} \cdot \bigcap_{x \in D_{e}}\left(\left(\{|M x|\}^{\downarrow} \rightarrow P x\right) \cap\left(\{|W x|\}^{\downarrow} \rightarrow \rightarrow P x\right)\right)
\end{aligned}
$$

\subsubsection{Wh-phrases}

In inquisitive semantics, just as in possibility semantics, wh-phrases like who or which song are treated as GQs, as illustrated in the following examples. ${ }^{25}$

$$
\begin{aligned}
& \llbracket \text { who sings } \rrbracket=\llbracket \text { who } \rrbracket(\llbracket \text { sings } \rrbracket) \\
& =\left[\lambda P_{\langle e, T\rangle} \cdot \bigcup_{x \in D_{e}} P x\right]\left(\lambda x .\{|S x|\}^{\downarrow}\right) \\
& =\bigcup_{x \in D_{e}}\{|S x|\}^{\downarrow} \\
& =\left\{|S x| \mid x \in D_{e}\right\}^{\downarrow} \\
& =\left[\lambda P_{\langle e, T\rangle} \cdot \lambda Q_{\langle e, T\rangle} \cdot \bigcup_{x \in D_{e}}(P x \cap Q x)\right] \\
& \left(\lambda x .\{|S x|\}^{\downarrow}\right)\left(\lambda x .\{|P x|\}^{\downarrow}\right) \\
& =\bigcup_{x \in D_{e}}\left(\{|S x|\}^{\downarrow} \cap\{|P x|\}^{\downarrow}\right) \\
& =\bigcup_{x \in D_{e}}\{|S x| \cap|P x|\}^{\downarrow} \\
& =\left\{|S x| \cap|P x| \mid x \in D_{e}\right\}^{\downarrow} \\
& =\left\{|S x \wedge P x| \mid x \in D_{e}\right\}^{\downarrow}
\end{aligned}
$$

Note that these question meanings are downward-closed: they contain all propositions that hold enough information to resolve the issue raised by the question. This differs from the treatment of questions in alternative semantics and possibility semantics. However, the alternatives in our inquisitive question meanings, i.e., the maximal elements, still correspond precisely to the standard Hamblin alternatives for who- and which-questions. For instance:

$$
\operatorname{ALT}\left(\text { which song is playing) }=\left\{|S x \wedge P x| \mid x \in D_{e}\right\}\right.
$$

\footnotetext{
25 The treatment of which phrases specified here is close to the one proposed by Groenendijk and Stokhof (1984). This treatment is known to face a number of problems (for instance, it assigns the same meaning to which men are bachelors and which bachelors are men), but addressing these is beyond the scope of the present paper. The purpose of the fragment specified here is just to illustrate the workings of the proposed framework.
} 


\subsubsection{Uniform typing of quantifiers and wh-phrases}

In our inquisitive semantics fragment, GQs and wh-phrases share the same semantic type, $\langle\langle e, T\rangle, T\rangle$. As a consequence, wh-phrases inherit some of the properties that are traditionally reserved for GQs. As we will see, this uniform typing opens up novel and interesting ways of analysing certain constructions. At the same time, however, there are also clear-cut differences between GQs and wh-phrases, which are classically derived by assigning these items different types. The uniform typing in inquisitive semantics invites us to reconsider what exactly to regard as the source of those differences between GQs and wh-phrases.

A first consequence of the uniform typing is that, just like GQs, wh-phrases can be coordinated by means of generalized conjunction and disjunction. In the following computation for which starter and which main dish, we use the same strategy as in the previous example, construing the GQ-denotations as sets of property-proposition pairs.

$$
\begin{aligned}
& \text { 匹which starter and which main dish } \rrbracket \\
& \quad=\left\{\left\langle P_{\langle e, T\rangle}, p_{\langle s, t\rangle}\right\rangle \mid p \in \bigcup_{x \in D_{e}}\left(\{|S x|\}^{\downarrow} \cap P x\right) \text { and } p \in \bigcup_{x \in D_{e}}\left(\{|M x|\}^{\downarrow} \cap P x\right)\right\} \\
& \quad=\left\{\left\langle P_{\langle e, T\rangle}, p_{\langle s, t\rangle}\right\rangle \mid p \in \bigcup_{x \in D_{e}}\left(\{|S x|\}^{\downarrow} \cap P x\right) \cap \bigcup_{x \in D_{e}}\left(\{|M x|\}^{\downarrow} \cap P x\right)\right\} \\
& \quad=\lambda P_{\langle e, T\rangle} \cdot \bigcup_{x \in D_{e}}\left(\{|S x|\}^{\downarrow} \cap P x\right) \cap \bigcup_{x \in D_{e}}\left(\{|M x|\}^{\downarrow} \cap P x\right)
\end{aligned}
$$

It is correctly predicted that in order to resolve the issue raised by a question with a conjoined wh-phrase, such as (51) below, one needs to pick a starter and a main dish, which amounts to resolving the issue raised by the conjoined question in (52).

Which starter and which main dish would you like?

Which starter would you like, and which main dish would you like?

We straightforwardly derive the same denotations for these two questions, without having to assume ellipsis in the first.

Similarly, an interrogative with a disjunctive wh-phrase such as (53) receives the same denotation as the disjunctive question in (54), reflecting the observation that both questions can be resolved by the same pieces of information: it suffices to pick a tea or to pick a coffee. ${ }^{26}$

Which tea or which coffee would you like?

Since wh-phrases and GQs have the same type, there is nothing in our semantic framework that prevents them from being coordinated, leading to apparent overgenerations such as *which man and every woman. This does not mean that a theory formulated in inquisitive semantics is inherently incapable of ruling such structures out, but rather that the explanatory burden has to be placed somewhere else than on the type system. We would like to suggest that this approach may in fact be advantageous, because the

\footnotetext{
26 For relevant discussion of disjoined wh-questions, see Szabolcsi (1997), Krifka (2001), Haida and Repp (2013) and Ciardelli et al. (2015).
} 
coordination of wh-phrases with bona-fide GQs can easily be ruled out on a syntactic basis, allowing for more fine-grained predictions than those made by a type-based account.

A widely-held assumption about the syntax of single wh-interrogatives in English is that the wh-item has to move to a functional head, typically to $\mathrm{C}$, in order to check its morphological [+wh]-feature (among many others, Chomsky 1977, 1995; Rizzi 2006). Since wh-movement out of a coordinated phrase is prohibited by island constraints (Ross 1967), structures like which $\operatorname{man}_{\boldsymbol{x}}$ did John see $\mathbf{t}_{\boldsymbol{x}}$ and every woman are ruled out. In contrast, if two wh-phrases are coordinated, the coordinated phrase as a whole bears a $[+w h]$-feature and can therefore also move as a whole, without violating island constraints.

What is more, the coordination of wh-phrases with bona-fide GQs is not as restricted as it appears at first sight. It becomes acceptable if the wh-item occurs in a quiz-master question or an echo question such as (55).

John saw WHICH man and every woman?

This fact can be explained in our framework, provided that wh-movement is not obligatory in these kinds of questions in English, while it cannot directly be explained in a framework that assumes different types for GQs and wh-phrases. ${ }^{27}$

A second characteristic that wh-phrases share with GQs in our system is their ability to undergo quantifier raising and to act as binders. Examples like (56) below, which Shan (2004) pointed out to be problematic for alternative semantics (see the discussion on page 4), can be given a straightforward analysis, by letting which man quantifier-raise and trigger lambda abstraction over the verb phrase. ${ }^{28}$

Which $\operatorname{man}_{x}$ sold which of his $x$ paintings?

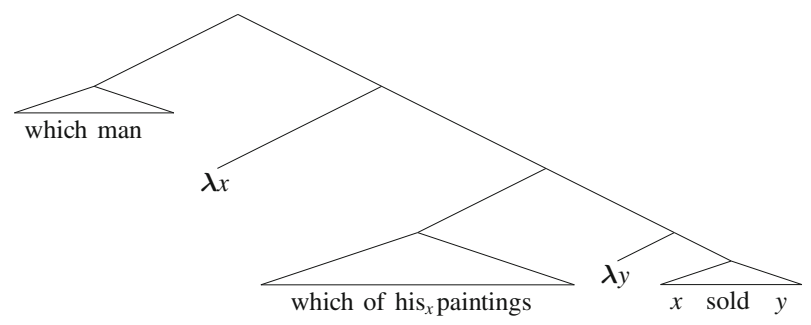

\footnotetext{
27 In a classically typed system, it may of course be stipulated that wh-phrases can be type-shifted in order to allow for coordination in the case of echo questions. But then the fact that coordination is ungrammatical in regular questions would have to be explained in a different way.

${ }^{28}$ For simplicity, his $_{\mathbf{X}}$ paintings is treated as a singular relational noun, where $P x y$ is to be read as $y$ is a painting by $x$.
} 


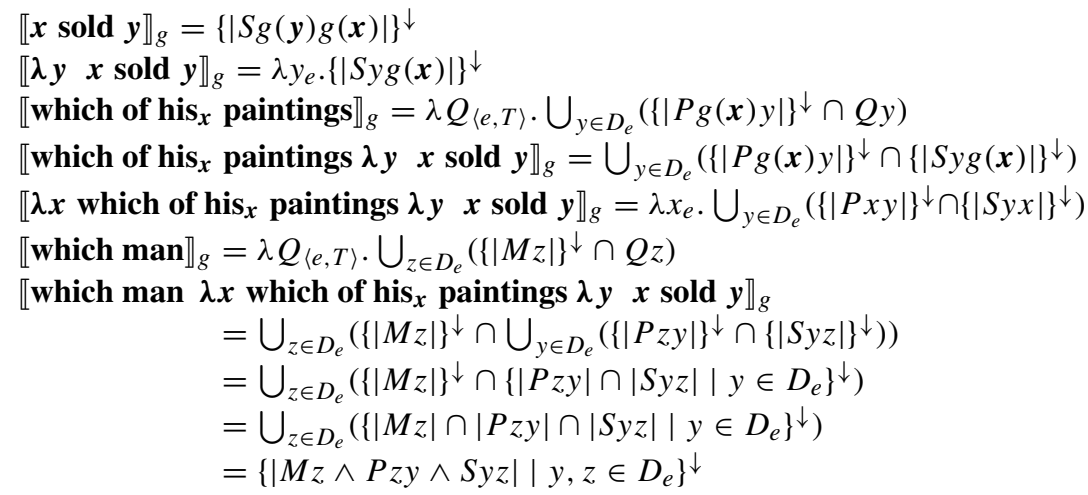

The third and final possibility that arises from the uniform typing of GQs and whphrases is a novel way of deriving pair-list readings for questions like (59).

What did everyone bring for the potluck?

This question has a salient interpretation, called the pair-list reading, on which it is understood as inquiring, for every person, what she brought for the potluck (see, e.g., Groenendijk and Stokhof 1984; Higginbotham 1991; Chierchia 1993; Szabolcsi 1997). In our framework, this reading can be derived simply by letting the universal outscope the wh-phrase. In a classically typed system, this would be compositionally impossible, whereas here it is unproblematic since interrogatives have the same type as declaratives and, again, wh-phrases have the same type as GQs. The pair-list reading is hence construed as universal quantification ranging over individuals and having scope over a question, or, in other words, as a large conjunction of questions, one for each individual: what $\operatorname{did} a$ bring, and what $\operatorname{did} b$ bring, and what did $c$ bring, ...? In order to resolve the issue raised by the pair-list question, one has to resolve all issues that are raised by the conjoined questions. To see what this amounts to formally, consider the derivation below for (59).

(60)

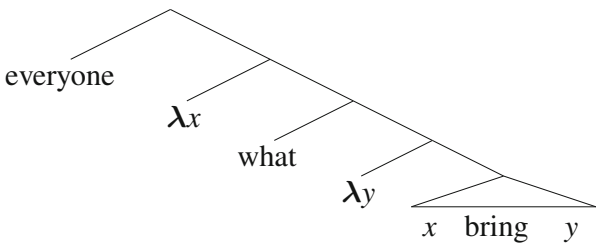




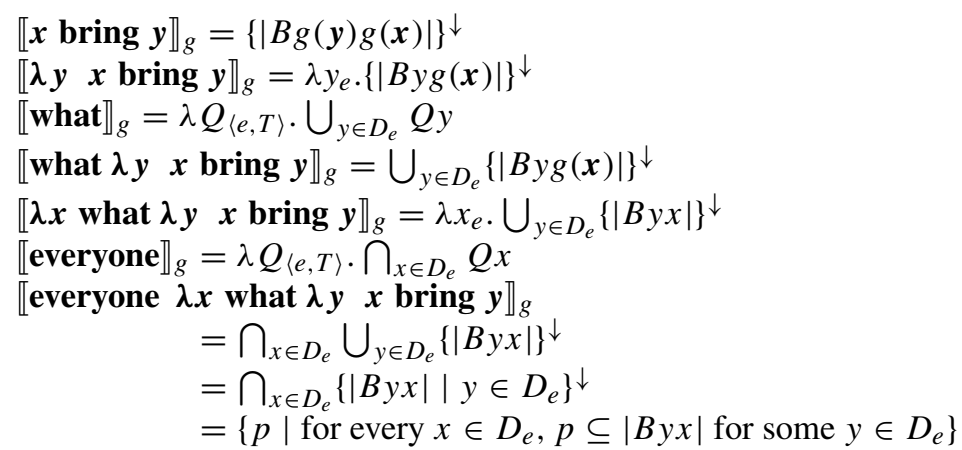

Hence, we can observe again that uniform typing removes constraints that, in a classically typed system, would arise as an immediate consequence of the type difference between GQs and wh-phrases. As we have already seen in the case of conjoined GQs and wh-phrases, however, there are two sides to this new-gained flexibility, with the danger of overgeneration never too far away. In the case of pair-list readings, on the one hand, we gain the ability to derive these readings in a very natural and transparent fashion, but on the other hand, we now have to find an explanation for those cases in which pair-list readings are blocked. To begin with, not all GQs allow such readings. While everybody lends itself naturally to a pair-list interpretation, for, e.g., most this reading seems to be blocked in matrix questions such as (62), though available if the question is embedded under an extensional verb, as in (63) (Szabolcsi 1997).

What did most people bring for the potluck?

Furthermore, there is an asymmetry between GQs occuring in subject position and in object position: the former readily allow pair-list readings, while for the latter these readings are much harder to come by (May 1985; Achimova et al. 2010). A detailed account of these phenomena lies beyond the scope of the current paper. ${ }^{29}$ The discussion here is merely meant to highlight the possibilities that arise from uniform typing.

Summing up this section then, we have seen that the issues with entailment and coordination that arise in alternative semantics may be avoided by reconsidering one of the basic features of the framework, namely, the identification of the meaning of a sentence with the set of alternatives that it generates. By teasing the two notions apart, construing the meaning of a sentence as a downward closed set of propositions, and viewing the maximal elements of this set as the alternatives that the sentence generates, we obtain a semantic framework which allows us to retain on the one hand an alternative-inducing notion of meaning, and on the other hand, the classical typetheoretic account of generalized entailment and coordination. ${ }^{30}$

\footnotetext{
29 Interestingly, the fact that quantifiers like most cannot directly scope over questions, unlike quantifiers like every, may be connected to the fact that, in the verbal domain, verbs like believe cannot apply to questions, unlike verbs like know. While these empirical contrasts have so far only been considered in isolation, inquisitive semantics may make it possible to give a unified explanation.

30 A brief note on how focus may be treated in our framework, to avoid possible confusion. We are not suggesting that focus semantic values also have to be downward closed sets of propositions, just like
} 


\section{Conclusion}

While it clearly seems that alternatives have an important role to play in semantics, the specific architecture of Hamblin-style alternative semantics forces us to give up two crucial features of classical compositional semantics, namely, (i) the typetheoretic composition operations of function application and abstraction and (ii) the type-theoretic treatment of cross-categorical entailment and coordination. This leads to a number of problems, both empirical and theoretical.

We have tried to identify precisely which features of alternative semantics are responsible for these issues, and how they could be modified in order to avoid the resulting problems. First, we argued that the compositionality issue stems from the assumption that all expressions denote sets of objects of the corresponding type. This assumption does not seem to have strong conceptual or empirical motivation, and dropping it does not seem to undermine the empirical coverage of the theories that are formulated in alternative semantics. This step led us to the framework of possibility semantics, where sentences still denote sets of propositions, but meanings are composed by means of the standard type-theoretic operations.

However, like alternative semantics, possibility semantics still faces the entailment issue, which also leads to problems in the treatment of conjunction. We argued that this issue stems from the assumption that the meaning of a sentence is identical with the set of alternatives that it generates. Once again, this assumption does not seem strictly necessary, neither from a conceptual point of view nor from an empirical one. Conceptually, there is another natural perspective on sentential meanings, motivated in recent work on inquisitive semantics, under which they are construed as sets of propositions that are downward closed. Empirically, all that is required for concrete linguistic applications of the framework is that the meaning of a sentence determine a set of alternatives. If the meaning of a sentence is a downward closed set of propositions, the maximal elements of this set are naturally viewed as the alternatives that the sentence generates. This conception of sentence meanings resolves the entailment issue: the general type-theoretic notion of entailment is recovered, and conjunction and disjunction can again be treated as meet and join operations w.r.t. entailment.

The resulting framework retains a fine-grained notion of meaning, which associates every sentence with a set of alternatives, but has a much more solid type-theoretic foundation than Hamblin-style alternative semantics: meanings are composed by means of the standard type-theoretic composition rules, compared by means of the standard notion of entailment as meaning inclusion, and coordinated by means of standard

\section{Footnote 30 continued}

ordinary sentential meanings. Rather, the most natural way to deal with focus in our framework would be to let the focus semantic value of an expression be, as usual, a set of objects of the same type as the ordinary semantic value of that expression. For instance, the focus semantic value of a proper name is a set of individuals, and the focus semantic value of a complete sentence is a set of inquisitive meanings, i.e., a set of downward closed sets of propositions. This is just the standard treatment of focus (Rooth 1985), only the types of objects that certain expressions receive as their ordinary semantic value have changed, and therefore the types of objects that they are assigned as their focus semantic value change accordingly. In principle, focus semantic values may be composed in a pointwise fashion, as in Rooth (1985). As far as we can see, the problems that we pointed out in Sect. 2 do not directly apply at the level of focus semantic values. 
generalized conjunction and disjunction. In this way, the empirical coverage of the analyses formulated in alternative semantics is preserved, while the observed framework issues are avoided.

Open Access This article is distributed under the terms of the Creative Commons Attribution 4.0 International License (http://creativecommons.org/licenses/by/4.0/), which permits unrestricted use, distribution, and reproduction in any medium, provided you give appropriate credit to the original author(s) and the source, provide a link to the Creative Commons license, and indicate if changes were made. 


\section{Appendix 1: Pointwise propagation of alternatives as a device for scope- taking}

A well-known problem in semantics is the observation that indefinite phrases have the ability to take exceptional wide scope (Farkas 1981; Fodor and Sag 1982). For instance, under a salient reading of (64), some relative of mine takes scope over the conditional (Reinhart 1997). This reading cannot be obtained if scope is determined purely by the LF configuration, since island constraints prevent the indefinite phrase from moving out of the antecedent.

If some relative of mine dies, I will inherit a house.

Kratzer and Shimoyama (2002), drawing inspiration from indeterminate pronouns in Japanese, demonstrate how exceptional scope can be derived as a consequence of composing alternatives via pointwise application: the alternative-generating phrase is interpreted in-situ, and the alternatives it introduces percolate upwards in the tree until they encounter an operator that "binds" them, such as existential closure or negation. It is clear that this alternative percolation is insensitive to island boundaries, and can therefore capture the exceptional scoping behaviour we observe with indefinites.

In contrast, in the framework proposed here pointwise propagation of alternatives cannot be used as a device for scope-taking. Since the mode of composition is classical function application, alternatives do not freely percolate upwards. The system could of course be extended to include an account of exceptional scope, and various options seem compatible with our approach (e.g., Jäger 2007; Brasoveanu and Farkas 2011; Onea 2015)_but exceptional scope cannot arise as a consequence of pointwise propagation of alternatives.

We believe, however, that this limitation does not present an argument against our framework, because we think that letting alternatives do double-duty as a device for scope-taking and modeling e.g. the semantics of questions is in fact problematic.

One problem for the double-duty strategy comes from the realm of embedded interrogatives. Since Hamblin (1973) and Karttunen (1977), it has been one of the standard approaches in question semantics to represent the meaning of interrogatives, both in root and in embedded contexts, as sets of alternatives. If alternatives are additionally used for scope-taking though, then it seems that these different functions can easily get confounded. For an example of this happening, consider the following sentence, where the if-clause contains an embedded interrogative which in turn contains an indefinite inside a scope island.

If John finds out which movies by a famous director are playing at the festival, he'll let us know.

It is not possible to derive the exceptional wide scope reading for the indefinite using pointwise propagation of alternatives as a scoping device, for the following reason. There are two alternative-generating expressions in the antecedent, the wh-phrase and the indefinite phrase. The indefinite phrase cannot leave its base position in the island, meaning that the alternatives from both sources have to be generated and composed within the scope of the question-embedding verb find out. Furthermore, they are 
composed by pointwise application, resulting in a flat set of alternatives that holds no information about the source of the alternatives:

$$
\{\operatorname{playing}(x) \mid \operatorname{movie}-\operatorname{by}(y, x), \operatorname{director}(y)\}
$$

This set is passed on in the derivation and subsequent operators have no way of distinguishing between the "alternativehood" introduced by the wh-phrase and that introduced by the indefinite. Such operators could be quantifiers (this has been brought to attention by Charlow (2014) and we will discuss it below) or, as here, elements responsible for the semantics of question-embedding. Extensional questionembedding predicates like find out are usually taken to express a relation between the subject and some answer to the embedded question. Hence, in the derivation of (65), an answer needs to be computed from the alternative set denoted by the question. ${ }^{31}$ That means that the question denotation, an alternative set, is turned into a proposition and only this proposition can be passed on to the further derivation steps. For the indefinite, this means that its scope is trapped inside the complement clause.

The reason that classical alternative semantics is unable to deal with sentences like (65) seems to be that the alternative sets it operates with are lacking structure. Charlow (2014) proposes a modified alternative semantics that makes it possible to treat the propagation of alternatives in a much more controlled fashion. In particular, alternative sets can be nested, permitting him to distinguish between different sources of alternatives and, thereby, to handle selective scope configurations. It is conceivable that, in Charlow's system, the answer operator would only apply to the inner layer of alternatives whereas the outer layer would be passed on in the computation, hence allowing for an account of cases like (65).

We take the picture so far to indicate that either (i) scope-taking and question semantics should not both be treated using alternatives, or (ii), if both scope-taking and question semantics are treated using alternatives, then these alternatives have to come with a richer structure and an overall compositional architecture that is more differentiated than unrestrained pointwise application.

Now, if scope-taking and question semantics should not both be dealt with in terms of the same mechanism, either of them stands in need of a different treatment. In our framework, alternatives are used to model the meaning of questions, but one may wonder whether the other choice is also viable: if question semantics were to be captured in a different way, would pointwise propagation of alternatives be a suitable tool for scope-taking? We believe that the answer is negative.

Let us first consider an argument by Charlow (2014) to the effect that classical alternative semantics is not capable of accounting for selective binding, e.g., for configurations in which multiple indefinites appear together in the same environment and only one of them takes exceptional wide scope. In particular, Charlow holds that in classical alternative semantics we cannot derive the reading of (66-a) in which a rich relative of mine takes exceptional scope without a favorable will doing so as well, and similarly for (66-b).

\footnotetext{
31 Whether it is the embedding predicate itself that computes this answer or some designated answer operator is immaterial in this context.
} 
(66) a. If a rich relative of mine draws up a favorable will, I'll inherit a house.

b. If a lawyer visits a rich relative of mine, I'll inherit a house.

The reason behind this limitation is the same as what we observed for example (65): alternative sets lack the structure to distinguish between alternatives from different sources. Therefore, an alternative-evaluating operator cannot single out a certain subset of alternatives; either all alternatives get bound, or none. However, while these examples illustrate the inability of alternative semantics to bind selectively, they don't seem sufficient to show that this framework fails as a general theory of scope-taking. This is because, in principle, configurations like those needed for (66) can be obtained in a nonselective framework if we relax the distribution of the existential closure operator. Standardly, this operator only applies to sentential constituents, hence necessarily binding all alternatives generated within the respective sentence. In contrast, if it could apply locally to a will or a lawyer, as sketched in (67), then the alternatives introduced by these phrases would be bound at a suitably low position. A second existential closure applying at sentence level would in turn bind the alternatives introduced by a relative of mine. ${ }^{32}$ Hence, at first sight it appears that, if we let existential closure apply locally enough, we can achieve the desired effect, overcoming Charlow's challenge.
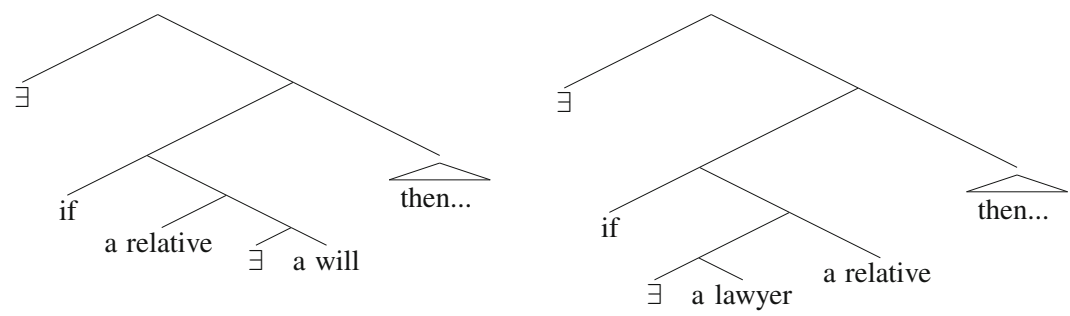

However, there are also scope configurations that evade this strategy. In (68) for example, one of the indefinites takes exceptional wide scope while the other oneand this is different from (67)-takes intermediate scope. Here, a professor cannot be bound by a locally applying existential closure operator since this would give it scope below every book. Placing the existential closure higher in the structure, such as above every book, is not an option either since this would bind the alternatives from both indefinite phrases.

Background: There is a course on medieval history at University X, which is not always taught by the same professor. All professors who have taught the course, though, managed to make most of their students very enthusiastic about the topic. So much so that, every year, most students who took the course read every book that their professor recommended.

\footnotetext{
32 Of course, the distribution of the existential closure operator would also need to be appropriately restricted, and this is not a trivial problem.
} 
Most students read every book that a professor recommended for a course on medieval history at University X.

Intended reading: a course $>$ most students $>$ a professor $>$ every book

This example shows that pointwise propagation of alternatives as implemented in classical alternative semantics cannot deal with selective binding and is therefore not a suitable basis for a general theory of scope-taking. In contrast, certain non-alternativebased theories of exceptional scope such as Brasoveanu and Farkas (2011) or Onea (2015) can capture the configuration in (68).

Charlow's enriched alternative semantics is able to deal with this example too, owing to two key characteristics of his system. First, as already mentioned, alternative sets can be nested, allowing for selective quantification. Second, the default mode of composition is standard function application; pointwise application only enters the picture through the semantics of certain type-shifting operators that, in effect, simulate pointwise application. This way, Charlow can employ pointwise application in a more controlled way, which gives him a handle on where in the derivation alternatives percolate and where they don't. Thus, although in Charlow's system scope arises partly through alternative percolation, his framework is fundamentally different from classical alternative semantics.

We have seen, then, that classical alternative semantics is unfit as a general theory of scope: trouble can arise when alternatives are simultaneously used for scope-taking and for question semantics, but also when they serve no such double function. We conclude that any theory which employs alternatives for scope-taking — regardless for what else it uses alternatives_-needs a richer structure for these alternatives and a way to curb their unrestrained propagation.

\section{References}

Achimova, A., Deprez, V., \& Musolino, J. (2010). What makes pair list answers available: An experimental approach. In NELS 41 Proceedings. United States: University of Pennsylvania.

Aloni, M. (2007). Free choice, modals and imperatives. Natural Language Semantics, 15(1), 65-94.

Alonso-Ovalle, L. (2006). Disjunction in alternative semantics. Ph.D. thesis, University of Massachusetts, Amherst.

Brasoveanu, A., \& Farkas, D. F. (2011). How indefinites choose their scope. Linguistics and Philosophy, $34,1-55$.

Champollion, L. (2016). Ten men and women got married today: Noun coordination and the intersective theory of conjunction. Journal of Semantics, 33(3), 561-622.

Charlow, S. (2014). On the semantics of exceptional scope. Ph.D. thesis, New York University.

Chierchia, G. (1993). Questions with quantifiers. Natural Language Semantics, 1(2), 181-234.

Chierchia, G., Fox, D., \& Spector, B. (2009). Hurford's constraint and the theory of scalar implicatures: Evidence for embedded implicatures. Presuppositions and implicatures (pp. 47-62). Cambridge, MA: MIT Press.

Chomsky, N. (1977). On wh-movement. In P. Culicover, A. Akmajian \& T. Wasow (Eds.), Formal syntax (pp. 71-132). New York: Academic Press.

Chomsky, N. (1995). The minimalist program (Vol. 1765). Cambridge: Cambridge University Press.

Ciardelli, I., \& Roelofsen, F. (2015). Alternatives in Montague grammar. In E. Csipak \& H. Zeijlstra (Eds.), Proceedings of Sinn und Bedeutung, Göttingen, pp. 161-178.

Ciardelli, I. \& Roelofsen, F. (2016). Hurford's constraint, the semantics of disjunctions, and the nature of alternatives. Manuscript, available on the Semantics Archive. 
Ciardelli, I., Groenendijk, J., \& Roelofsen, F. (2013). Inquisitive semantics: A new notion of meaning. Language and Linguistics Compass, 7(9), 459-476.

Ciardelli, I., Groenendijk, J., \& Roelofsen, F. (2015). Inquisitive semantics. ESSLLI lecture notes. Available via www.illc.uva.nl/inquisitivesemantics.

Farkas, D. (1981). Quantifier scope and syntactic islands. Proceedings of CLS, 7, 59-66.

Fodor, J. D., \& Sag, I. A. (1982). Referential and quantificational indefinites. Linguistics and Philosophy, 5, 355-398.

Groenendijk, J. \& Stokhof, M. (1984). Studies on the semantics of questions and the pragmatics of answers. Ph.D. thesis, University of Amsterdam.

Hagstrom, P. A. (1998). Decomposing questions. Ph.D. thesis, MIT.

Haida, A. \& Repp, S. (2013). Disjunction in wh-questions. In Proceedings of NELS 40.

Hamblin, C. L. (1973). Questions in Montague English. Foundations of Language, 10(1), 41-53.

Higginbotham, J. (1991). Interrogatives I. In L. Cheng \& H. Demirdash (Eds.), More papers on whmovement. MIT Working Papers in Linguistics.

Hurford, J. (1974). Exclusive or inclusive disjunction. Foundations of Language, 11(3), 409-411.

Jacobson, P. (1999). Towards a variable-free semantics. Linguistics and Philosophy, 22(2), 117-185.

Jäger, G. (2007). Partial variables and specificity. In U. Sauerland \& P. Stateva (Eds.), Presupposition and implicature in compositional semantics (pp. 121-162). New York: Palgrave Macmillan.

Karttunen, L. (1977). Syntax and semantics of questions. Linguistics and Philosophy, 1, 3-44.

Katzir, R., \& Singh, R. (2013). Hurford disjunctions: Embedded exhaustification and structural economy. Proceedings of Sinn und Bedeuting, 18, 201-216.

Keenan, E. L., \& Faltz, L. M. (1985). Boolean semantics for natural language. New York: Springer.

Kratzer, A. \& Shimoyama, J. (2002). Indeterminate pronouns: The view from Japanese. In Y. Otsu (Ed.), Proceedings of the third Tokyo conference on psycholinguistics, pp. 1-25.

Krifka, M. (2001). Quantifying into question acts. Natural Language Semantics, 9(1), 1-40.

May, R. (1985). Logical Form: Its structure and derivation (Vol. 12). Cambridge, MA: MIT Press.

Menéndez-Benito, P. (2005). The grammar of choice. Ph.D. thesis, University of Massachusetts, Amherst.

Montague, R. (1970). Universal grammar. Theoria, 36(3), 373-398.

Montague, R. (1973). The proper treatment of quantification in ordinary English. In J. Hintikka, J. Moravcsik, \& P. Suppes (Eds.), Approaches to natural language (pp. 221-242). Dordrecht: Springer.

Novel, M., \& Romero, M. (2010). Movement, variables and Hamblin alternatives. Proceedings of Sinn und Bedeutung, 14, 322-338.

Onea, E. (2015). Why indefinites can escape scope islands. Linguistics and Philosophy, 38(3), 1-31.

Partee, B. H., \& Rooth, M. (1983). Generalized conjunction and type ambiguity. In R. Bäuerle, C. Schwarze, \& A. von Stechow (Eds.), Meaning, use and interpretation of language (pp. 115-143). Berlin: De Gruyter.

Poesio, M. (1996). Semantic ambiguity and perceived ambiguity. In K. van Deemter \& S. Peters (Eds.), Semantic ambiguity and underspecification (pp. 231-250). Stanford, CA: CSLI.

Rawlins, K. (2008). (Un)conditionals: an investigation in the syntax and semantics of conditional structures. Ph.D. thesis, University of California, Santa Cruz.

Reinhart, T. (1997). Quantifier scope: How labor is divided between QR and choice functions. Linguistics and Philosophy, 20(4), 335-397.

Rizzi, L. (2006). On the form of chains: Criterial positions and ECP effects. In L. Cheng \& N. Corver (Eds.), Wh-movement: Moving on (pp. 97-134). Cambridge, MA: MIT Press.

Roelofsen, F. (2013). Algebraic foundations for the semantic treatment of inquisitive content. Synthese, 190(1), 79-102.

Roelofsen, F. (2015). Two alternatives for disjunction: an inquisitive reconciliation. Manuscript, submitted for publication. Available via www.illc.uva.nl/inquisitivesemantics.

Roelofsen, F. \& van Gool, S. (2010). Disjunctive questions, intonation, and highlighting. In M. Aloni, H. Bastiaanse, T. de Jager, \& K. Schulz (Eds.), Logic, language, and meaning: Selected papers from the seventeenth Amsterdam colloquium (pp. 384-394). New York: Springer.

Rooth, M. (1985). Association with focus. Ph.D. thesis, University of Massachusetts, Amherst.

Ross, J. R. (1967). Constraints on variables in syntax. Ph.D. thesis, Massachusetts Institute of Technology.

Shan, C.-C. (2004). Binding alongside Hamblin alternatives calls for variable-free semantics. In Proceedings of semantics and linguistic theory (SALT 14) (pp. 289-304).

Shimoyama, J. (2001). Wh-constructions in Japanese. Ph.D. thesis, University of Massachusetts, Amherst. 
Shimoyama, J. (2006). Indeterminate phrase quantification in Japanese. Natural Language Semantics, 14(2), $139-173$.

Simons, M. (2005). Dividing things up: The semantics of or and the modal/or interaction. Natural Language Semantics, 13(3), 271-316.

Szabolcsi, A. (1987). Bound variables in syntax: Are there any? In Proceedings of the 6th Amsterdam colloquium (pp. 331-353).

Szabolcsi, A. (1997). Quantifiers in pair-list readings. In A. Szabolcsi (Ed.), Ways of scope taking (pp. 311-347). New York: Springer.

Theiler, N. (2014). A multitude of answers: Embedded questions in typed inquisitive semantics. MSc thesis, University of Amsterdam.

von Stechow, A. (1991). Focusing and backgrounding operators. In W. Abraham (Ed.), Discourse particles: Descriptive and theoretical investigations on the logical, syntactic and pragmatic properties of discourse particles in German (pp. 37-84). Amsterdam: John Benjamins.

Winter, Y. (2001). Flexibility principles in Boolean semantics: The interpretation of coordination, plurality, and scope in natural language. Cambridge, MA: MIT Press. 